\title{
UPPER BOUNDS ON PROBABILITY THRESHOLDS FOR ASYMMETRIC RAMSEY PROPERTIES
}

\author{
YOSHIHARU KOHAYAKAWA, MATHIAS SCHACHT, AND RETO SPÖHEL
}

\begin{abstract}
Given two graphs $G$ and $H$, we investigate for which functions $p=p(n)$ the random graph $G_{n, p}$ (the binomial random graph on $n$ vertices with edge probability $p$ ) satisfies with probability $1-o(1)$ that every red-blue-coloring of its edges contains a red copy of $G$ or a blue copy of $H$. We prove a general upper bound on the threshold for this property under the assumption that the denser of the two graphs satisfies a certain balancedness condition. Our result partially confirms a conjecture by the first author and Kreuter, and together with earlier lower bound results establishes the exact order of magnitude of the threshold for the case in which $G$ and $H$ are complete graphs of arbitrary size.

In our proof we present an alternative to the so-called deletion method, which was introduced by Rödl and Ruciński in their study of symmetric Ramsey properties of random graphs (i.e. the case $G=H$ ), and has been used in many proofs of similar results since then.
\end{abstract}

\section{§1. INTRODUCTION}

1.1. Ramsey properties of random graphs. Ramsey properties of random graphs were studied first by Frankl and Rödl [6], and much effort has been devoted to their further investigation since then. Perhaps most notably, Rödl and Ruciński [20,21] established a general threshold result that we present in the following.

For any two graphs $F$ and $H$, let

$$
F \rightarrow(H)_{k}
$$

denote the property that every edge-coloring of $F$ with $k$ colors contains a monochromatic copy of $H$. Throughout, we denote the number of edges and vertices of a graph $G$ by $e_{G}$

The first authors was partially supported by CNPq (Proc. 308509/2007-2 and Proc. 484154/2010-9). The collaboration of the first two authors was supported by a CAPESÂUUDAAD collaboration grant. The second author was supported through the Heisenberg-Programme of the Deutsche Forschungsgemeinschaft (DFG Grant SCHA 1263/4-1). The third author was supported by a grant from the Swiss National Science Foundation and the research was partially carried out when the author was still at ETH Zurich. The authors are grateful to NUMEC/USP, Núcleo de Modelagem Estocástica e Complexidade of the University of São Paulo, for its hospitality. 
and $v_{G}$ respectively (sometimes also by $e(G)$ and $v(G)$ ). We say that a graph is nonempty if it has at least one edge. For any graph $H$ we define

$$
d_{2}(H):= \begin{cases}\frac{e_{H}-1}{v_{H}-2} & \text { if } v_{H} \geqslant 3 \\ 1 / 2 & \text { if } H \cong K_{2} \\ 0 & \text { if } e_{H}=0,\end{cases}
$$

and set

$$
m_{2}(H):=\max _{J \subseteq H} d_{2}(J) .
$$

We say that $H$ is 2-balanced if $m_{2}(H)=d_{2}(H)$, and strictly 2-balanced if in addition $m_{2}(H)>d_{2}(J)$ for all proper subgraphs $J \subsetneq H$. With the notation above, a slightly simplified version of the result of Rödl and Ruciński reads as follows. (The lower bound proof given in [20] does not cover the case where $J \subseteq H$ maximizing $d_{2}(J)$ is a triangle; however, this case was settled earlier in [18].)

Recall that in the binomial random graph $G_{n, p}$ on $n$ vertices, every edge is present with probability $0 \leqslant p=p(n) \leqslant 1$ independently of all other edges.

Theorem 1 (Rödl and Ruciński [20,21]). Let $k \geqslant 2$ and $H$ be a graph that is not a forest. Then there exist constants $c, C>0$ such that

$$
\lim _{n \rightarrow \infty} \mathbb{P}\left(G_{n, p} \rightarrow(H)_{k}\right)= \begin{cases}0 & \text { if } p=p(n) \leqslant c n^{-1 / m_{2}(H)} \\ 1 & \text { if } p=p(n) \geqslant C n^{-1 / m_{2}(H)}\end{cases}
$$

where $m_{2}(H)$ is defined in (1) and (2).

We will refer to the two statements made by Theorem 1 as the 0 - and the 1-statement, respectively, and to the function $p_{H}(n)=n^{-1 / m_{2}(H)}$ as the threshold for the Ramsey property $F \rightarrow(H)_{k}$. The 1-statement of Theorem 1 is also true when $H$ is any forest that is not a matching; for the 0-statement however there are a few well-understood nontrivial exceptions (see e.g. [11, Section 8.1]).

A vertex-coloring analogue of Theorem 1 was proved earlier in [18], and generalizations of Theorem 1 to the (uniform) hypergraph setting were studied in [7,22,23]. Most work on the hypergraph setting has focused on the corresponding 1-statements, i.e., on proving upper bounds on the thresholds of the respective Ramsey properties. This line of work has been settled quite recently by the results of [7], which imply 1-statements analogous to that of Theorem 1 for even more general settings. Similar results were reported by Conlon and Gowers [4]. 
1.2. Asymmetric Ramsey properties. In Theorem 1 the same graph $H$ is forbidden in every color class. In this paper we are concerned with the natural generalization of this setup where a different graph is forbidden in each of the $k$ color classes. Within classical Ramsey theory the study of these so-called asymmetric Ramsey properties led to many interesting questions and results; see e.g. [3].

For any graphs $F, H_{1}, \ldots, H_{k}$, let

$$
F \rightarrow\left(H_{1}, \ldots, H_{k}\right)
$$

denote the property that every edge-coloring of $F$ with $k$ colors contains a monochromatic copy of $H_{i}$ in color $i$ for some $1 \leqslant i \leqslant k$. The threshold of this asymmetric Ramsey property was determined for the case in which all the $H_{i}$ are cycles $C_{\ell_{i}}$ (here $C_{\ell}$ denotes the cycle of length $\ell$ ) by the first author and Kreuter.

Theorem $2([14])$. Let $k \geqslant 2$ and $3 \leqslant \ell_{1} \leqslant \cdots \leqslant \ell_{k}$ be integers. Then there exist constants c, $C>0$ such that

$$
\lim _{n \rightarrow \infty} \mathbb{P}\left(G_{n, p} \rightarrow\left(C_{\ell_{1}}, \ldots, C_{\ell_{k}}\right)\right)= \begin{cases}0 & \text { if } p=p(n) \leqslant c n^{-1 / m_{2}\left(C_{\ell_{2}}, C_{\ell_{1}}\right)} \\ 1 & \text { if } p=p(n) \geqslant C n^{-1 / m_{2}\left(C_{\ell_{2}}, C_{\ell_{1}}\right)}\end{cases}
$$

where

$$
m_{2}\left(C_{\ell_{2}}, C_{\ell_{1}}\right):=\frac{\ell_{1}}{\ell_{1}-2+\left(\ell_{2}-2\right) /\left(\ell_{2}-1\right)} .
$$

Note that the threshold does not depend on $\ell_{3}, \ldots, \ell_{k}$ in order of magnitude.

In the same paper, an explicit threshold function for asymmetric Ramsey properties involving arbitrary graphs $H_{i}$ is conjectured. The conjecture is stated for the two-color case, and also we will restrict our attention to this case in the following. We will briefly return to the case with more colors at the end of this paper.

For any two graphs $G$ and $H$ we let

$$
d_{2}(G, H):= \begin{cases}\frac{e_{H}}{v_{H}-2+1 / m_{2}(G)} & \text { if } e_{G}, e_{H} \geqslant 1 \\ 0 & \text { otherwise }\end{cases}
$$

(where $m_{2}(G)$ is defined in (1) and (2)), and set

$$
m_{2}(G, H):=\max _{J \subseteq H} d_{2}(G, J) .
$$

We say that $H$ is balanced w.r.t. $d_{2}(G, \cdot)$ if $m_{2}(G, H)=d_{2}(G, H)$, and strictly balanced w.r.t. $d_{2}(G, \cdot)$ if in addition $m_{2}(G, H)>d_{2}(G, J)$ for all proper subgraphs $J \subsetneq H$.

It can be verified that $m_{2}(G, G)=m_{2}(G)$ for any graph $G$ and, more generally, that for any two graphs $G$ and $H$ with $m_{2}(G) \leqslant m_{2}(H)$ we have $m_{2}(G) \leqslant m_{2}(G, H) \leqslant m_{2}(H)$, with both inequalities strict if $0<m_{2}(G)<m_{2}(H)$. The conjecture in [14] is as follows. 
Conjecture 3 ([14]). Let $G$ and $H$ be graphs that are not forests with $m_{2}(G) \leqslant m_{2}(H)$. Then there exist constants $c, C>0$ such that

$$
\lim _{n \rightarrow \infty} \mathbb{P}\left(G_{n, p} \rightarrow(G, H)\right)= \begin{cases}0 & \text { if } p=p(n) \leqslant c n^{-1 / m_{2}(G, H)} \\ 1 & \text { if } p=p(n) \geqslant C n^{-1 / m_{2}(G, H)}\end{cases}
$$

where $m_{2}(G, H)$ is defined in (3) and (4).

The assumption that $G$ and $H$ are not forests was not made in the original formulation of Conjecture 3, but without it the 0-statement fails to hold even in the symmetric case, as mentioned above.

The threshold function stated in Conjecture 3 can be motivated as follows. Let $G$ and $H$ be graphs with $0<m_{2}(G)<m_{2}(H)$, and assume that we are looking for a redblue-coloring of $G_{n, p}$ that contains no red copy of $G$ and no blue copy of $H$. For simplicity, suppose that $m_{2}(G)=\left(e_{G}-1\right) /\left(v_{G}-2\right)$ and $m_{2}(G, H)=e_{H} /\left(v_{H}-2+1 / m_{2}(G)\right)$. Note that w.l.o.g. we may assign color blue to all edges that are not contained in a copy of $H$ - in other words, only the edges of $G_{n, p}$ that are contained in copies of $H$ are relevant for the Ramsey property $G_{n, p} \rightarrow(G, H)$. We shall call these edges $H$-edges in the following. By standard calculations, for $p=c n^{-1 / m_{2}(G, H)}$ the expected number of $H$-edges in $G_{n, p}$ is of order $n^{v_{H}-e_{H} / m_{2}(G, H)}=n^{2-1 / m_{2}(G)}$, and if these edges behave like edges of a random graph $G_{n, p^{*}}$ with $p^{*}=c^{\prime} n^{-1 / m_{2}(G)}$, the expected number of copies of $G$ that are formed by such $H$-edges and contain a given edge of $G_{n, p}$ is a constant depending on $c$. If this constant is close to zero, the copies of $G$ formed by $H$-edges in $G_{n, p}$ should be loosely scattered, and we can color one edge blue in each of these copies without creating blue copies of $H$ in the process. On the other hand, if this constant is large, the copies of $G$ formed by $H$-edges of $G_{n, p}$ will highly intersect with each other, and, according to the conjecture, almost surely there will be no coloring avoiding both a red copy of $G$ and a blue copy of $H$.

The reader may wonder why a similar reasoning with the roles of $G$ and $H$ reversed is not equally justified. The reason is that whenever $p$ is larger than $n^{-1 / m_{2}(G)}$ by an appropriate polylogarithmic factor (in particular for $p=c n^{-1 / m_{2}(G, H)}$ as above), with high probability every edge of $G_{n, p}$ is contained in a copy of $G$. (Recall that $G$ is the sparser of the two graphs.) Thus the notion of ' $G$-edges' is meaningless in our context.

A vertex-coloring analogue of Conjecture 3 was proved by Kreuter [17]. The only significant progress towards proving Conjecture 3 since its publication in [14] concerns the 0 -statement, which was shown to hold for the case in which $G$ and $H$ are complete graphs of arbitrary fixed sizes in [19]. 
The approach employed in [14] for the proof of the 1-statement of Theorem 2 is based on the sparse version of Szemerédi's regularity lemma (see $[13,16])$. The approach via sparse regularity can be extended to prove the 1-statement of Conjecture 3 for any two graphs $G$ and $H$, provided the so-called KŁR-Conjecture [15] holds for $G$ and $H$ is strictly balanced w.r.t. $d_{2}(G, \cdot)$ (see [19]; additionally, Lemma 16 in [14] needs to be modified slightly to relax the condition on $H$ from '2-balanced' to 'strictly balanced w.r.t. $d_{2}(G, \cdot)$ '). The KŁR-Conjecture has been proven for cycles of arbitrary size, and for complete graphs on up to five vertices. For references and a comprehensive overview of the status quo of that conjecture, we refer to the survey article [8].

1.3. Our results. In this paper we prove the 1-statement of Conjecture 3 under the same balancedness assumption for $H$ as is needed for the approach via sparse regularity, but without invoking the KŁR-Conjecture for $G$. We say that a graph is a matching if it has maximum degree at most 1.

Theorem 4 (Main result). Let $G$ and $H$ be graphs that are not matchings such that $H$ is strictly balanced w.r.t. $d_{2}(G, \cdot)$. Then there exists a constant $C>0$ such that for $p=p(n) \geqslant C n^{-1 / m_{2}(G, H)}$ we have

$$
\lim _{n \rightarrow \infty} \mathbb{P}\left(G_{n, p} \rightarrow(G, H)\right)=1 .
$$

Recall that we suppose that $m_{2}(G) \leqslant m_{2}(H)$ in Conjecture 3. One can show that the assumption that $H$ should be strictly balanced w.r.t. $d_{2}(G, \cdot)$ in Theorem 4 implies that $m_{2}(G)<m_{2}(H)$.

There is an equivalent formulation of the hypothesis of strict balancedness in Theorem 4 . For every subgraph $J$ of $H$, let $\mu(J ; n, p)$ be the expected number of occurences of $J$ in $G_{n, p}$. Then $H$ is strictly balanced w.r.t. $d_{2}(G, \cdot)$ if and only if

$$
\mu\left(H ; n, n^{-1 / m_{2}(G, H)}\right)=o\left(\mu\left(J ; n, n^{-1 / m_{2}(G, H)}\right)\right)
$$

for every proper subgraph $J$ of $H$ (see Remark 12 and Lemma 13(ii) below).

Our proof of Theorem 4 does not use sparse regularity at all, and has in fact more in common with the original proof of the 1-statement for the symmetric case (Theorem 1), due to Rödl and Ruciński, than with the proof of Theorem 2 given in [14]. We believe that a feature of interest in our proof is that it introduces a different approach for handling certain technical difficulties that are dealt with in the Rödl-Ruciński proof via the so called 'deletion method' (for details, see Section 1.4).

Together with the lower bound results for complete graphs we already mentioned [19], our result establishes general threshold functions for the case where $G=K_{\ell}$ and $H=K_{r}$ are complete graphs of fixed sizes $\ell<r$. 
Corollary 5. Let $3 \leqslant \ell<r$ be integers. Then there exist constants $c, C>0$ such that

$$
\lim _{n \rightarrow \infty} \mathbb{P}\left(G_{n, p} \rightarrow\left(K_{\ell}, K_{r}\right)\right)= \begin{cases}0 & \text { if } p=p(n) \leqslant c n^{-1 / m_{2}\left(K_{\ell}, K_{r}\right)} \\ 1 & \text { if } p=p(n) \geqslant C n^{-1 / m_{2}\left(K_{\ell}, K_{r}\right)}\end{cases}
$$

where

$$
m_{2}\left(K_{\ell}, K_{r}\right)=\frac{\left(\begin{array}{l}
r \\
2
\end{array}\right)}{r-2+2 /(\ell+1)}
$$

We can use Theorem 4 to infer statements about the existence of locally sparse graphs $F$ that enjoy the asymmetric Ramsey property $F \rightarrow(G, H)$, similarly to those presented in [21] for symmetric Ramsey properties. We refrain from a general statement of these results, and only mention the following corollary, which is an asymmetric variant of Corollary 5 in [21] and can be deduced analogously.

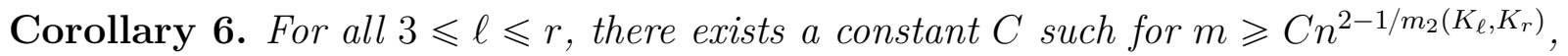
almost all graphs $F$ on $n$ vertices with $m$ edges that contain no copy of $K_{r+1}$ satisfy $F \rightarrow\left(K_{\ell}, K_{r}\right)$

We close with a deterministic consequence of Corollary 5. A graph $F$ is called Ramseycritical, or simply critical, for a pair of graphs $(G, H)$, if $F \rightarrow(G, H)$ but, for any proper subgraph $F^{\prime}$ of $F$, the relation $F^{\prime} \rightarrow(G, H)$ fails. The pair $(G, H)$ is called Ramsey-finite if the class $\mathcal{C}(G, H)$ of all graphs that are critical for $(G, H)$ is finite, and Ramsey-infinite otherwise. Note that, by definition, the Ramsey property $F \rightarrow(G, H)$ is equivalent to $F$ containing a copy of a graph from $\mathcal{C}(G, H)$.

The following result was originally proved by constructive means by Burr, Erdős, and Lovasz [2]. We obtain an alternative (non-constructive) proof as an immediate consequence of Corollary 5.

Corollary 7. For all $3 \leqslant \ell<r$, the pair $\left(K_{\ell}, K_{r}\right)$ is Ramsey-infinite.

Proof. It is well-known (and can be shown similarly to [11, Theorem 3.9]) that for any finite family $\mathcal{F}$, the property $\mathcal{P}=\mathcal{P}(\mathcal{F})$ of containing a copy of a graph from $\mathcal{F}$ admits a threshold $p_{\mathcal{F}}=p_{\mathcal{F}}(n)$ such that, for any constant $c>0$, the random graph $G_{n, p}$ with $p=c p_{\mathcal{F}}$ satisfies

$$
0<\liminf _{n \rightarrow \infty} \mathbb{P}\left(G_{n, p} \in \mathcal{P}\right) \leqslant \limsup _{n \rightarrow \infty} \mathbb{P}\left(G_{n, p} \in \mathcal{P}\right)<1
$$

Corollary 5 implies that the property $F \rightarrow\left(K_{\ell}, K_{r}\right)$ does not admit a function $p_{\mathcal{F}}=p_{\mathcal{F}}(n)$ and hence Corollary 7 follows. 
1.4. An alternative to the deletion method. Our proof of Theorem 4 reuses many ideas of the proof of the 1-statement of Theorem 1 given in [21]. However, we point out one particular technical issue that is solved in a quite different way from [21] in our proof. Namely, at some point in the proof one needs to control the upper tail of the random variable that counts the number of copies of some given graph $T$ in $G_{n, p}$.

In typical proofs of similar results (see, e.g., [7, 22-24]), this is taken care of by the so-called deletion method (see also [12]), i.e., by allowing the deletion of a small fraction of edges to get the desired exponentially small error probability. This is formalized in the 'deletion lemma' [21, Lemma 4] (see also [11, Lemma 2.51]).

This deletion lemma is then combined with a 'robustness lemma' [21, Lemma 3] (see also [11, Lemma 2.52]), which states that monotone properties (like the Ramsey properties discussed here) that hold with probability exponentially close to 1 continue to hold with similarly high probabilities even if an adversary is allowed to delete a small fraction of the edges. This robustness lemma is needed to guarantee that the few edges that were deleted to control the number of copies of $T$ do not destroy other properties that are important for the proof.

In our proof we use a different and arguably simpler approach to control the number of copies of $T$. Namely, we condition on the number of copies of $T$ in $G_{n, p}$ not being too large, and apply the Harris inequality [9] (Theorem 17) to show that this only increases the probability that other relevant properties fail to hold (and, hence, bounding the probability of such bad events in the conditional space from above gives upper bounds for the probability of those bad events in the original space). Thus we may work in the conditional space. The fact that the event on which we condition holds with reasonable probability (constant probability is more than enough here) implies that the conditional space we are considering behaves essentially like the original space, except that with probability 1 the number of copies of $T$ is not too large. Thus there is no need to delete edges in our approach. We believe that many of the earlier proofs in the field, in particular the proof

given in [21] for the symmetric case (Theorem 1), can be simplified analogously from the technical point of view.

1.5. Organization of this paper. We collect a number of definitions and auxiliary statements in Section 2, and prove Theorem 4 in Section 3. We discuss possible extensions of our results in Section 4.

\section{§2. Preliminaries}

2.1. Basic inequalities. We begin by stating some equalities that follow immediately from the definitions of $m_{2}(G)$ and $m_{2}(G, H)$, and that will be used throughout this paper. 
Recall that we call a graph nonempty if it has at least one edge. The definitions in (1) and (2) imply that for any nonempty graph $G$ and any subgraph $I \subseteq G$ with $v_{I} \geqslant 2$ we have

$$
v_{I}-\frac{e_{I}}{m_{2}(G)} \geqslant 2-\frac{1}{m_{2}(G)}
$$

(with equality for $I=G$ if $G$ is 2-balanced). Similarly, the definitions in (3) and (4) imply that for any two nonempty graphs $G$ and $H$ and any subgraph $J \subseteq H$ with $v_{J} \geqslant 2$ we have

$$
v_{J}-\frac{e_{J}}{m_{2}(G, H)} \geqslant 2-\frac{1}{m_{2}(G)}
$$

(with equality for $J=H$ if $H$ is balanced w.r.t. $d_{2}(G, \cdot)$ ). Combining the previous two equalities yields in particular that for any two nonempty graphs $G$ and $H$ we have

$$
v_{G}-2+\left(e_{G}-1\right)\left(v_{H}-2\right)-\frac{\left(e_{G}-1\right) e_{H}}{m_{2}(G, H)} \stackrel{(6)}{\geqslant} v_{G}-2-\frac{e_{G}-1}{m_{2}(G)} \stackrel{(5)}{\geqslant} 0,
$$

which will become important later on.

2.2. $H$-covered copies. The following definitions will be crucial in our inductive scheme.

Definition 8. For graphs $H$ and $A$, we denote by $E_{H}(A) \subseteq E(A)$ the union of the edge sets of all copies of $H$ in $A$. We will refer to the edges in $E_{H}(A)$ as the $H$-edges of $A$. Furthermore, we say that a copy $\bar{G}$ of a graph $G$ in $E_{H}(A)$ is $H$-covered in $A$ if there is a family of $e_{G}$ pairwise edge-disjoint copies of $H$ in $A$ such that each edge of $\bar{G}$ is contained in (exactly) one of these copies.

Note that not every copy of $G$ that is formed by $H$-edges of $A$ is $H$-covered in $A$.

Definition 9. For any two graphs $G$ and $H$, let $\mathcal{F}(G, H)$ denote the family of all graphs obtained by taking a copy of $G$ and embedding each of its edges into a copy of $H$ such that these $e_{G}$ copies of $H$ are pairwise edge-disjoint (not nessarily vertex-disjoint).

We denote the graphs in $\mathcal{F}(G, H)$ by $G^{H}$, and refer to a copy of $G$ in $G^{H}$ that can be used to construct $G^{H}$ as described as a central copy of $G$ in $G^{H}$ (in general, for a given $G^{H} \in \mathcal{F}(G, H)$ such a central copy is not uniquely defined). Note that a copy of $G$ in some graph $A$ is $H$-covered if and only if it is a central copy in a copy of some graph $G^{H} \in \mathcal{F}(G, H)$ in $A$.

For any $G$ and $H$ and any graph $G^{H} \in \mathcal{F}(G, H)$, let

$$
L\left(G^{H}\right):=v_{G}+e_{G} \cdot\left(v_{H}-2\right)-v\left(G^{H}\right) \geqslant 0 .
$$


Intuitively, this quantity denotes the number of vertices that are 'lost' because the copies of $H$ forming $G^{H}$ intersect in more vertices than specified by $G$. Thus we have

$$
\begin{aligned}
& e\left(G^{H}\right)=e_{G} \cdot e_{H}, \\
& v\left(G^{H}\right)=v_{G}+e_{G} \cdot\left(v_{H}-2\right)-L\left(G^{H}\right) .
\end{aligned}
$$

Our induction is on the number of edges of $G$, and we will mostly need the above definitions for a certain graph $G_{-}$with $e(G)-1$ edges to which we apply the induction hypothesis. The following technical lemma will become important later on.

Lemma 10. Let $G$ be a graph that is not a matching, let $H$ be a nonempty graph, and fix some subgraph $G_{-} \subseteq G$ with $e\left(G_{-}\right)=e(G)-1$ and $v\left(G_{-}\right)=v(G)$. Furthermore, let a graph $G_{-}^{H} \in \mathcal{F}\left(G_{-}, H\right)$ with central copy $G_{-}^{\prime}$ be given, and let $g$ denote a vertex pair that completes $G_{-}^{\prime}$ to a copy of $G$ when inserted as an edge. Then every subgraph $J \subseteq G_{-}^{H}$ that contains the two vertices of $g$ satisfies

$$
v(J)-\frac{e(J)}{m_{2}(G, H)} \geqslant 2-L\left(G_{-}^{H}\right) .
$$

Proof. Note that it suffices to prove the claim for induced subgraphs $J \subseteq G_{-}^{H}$. We consider a fixed such subgraph $J$ and decompose it as follows. Let $E^{\prime}:=E\left(G_{-}^{\prime}\right)$ denote the edge set of the central copy $G_{-}^{\prime}$. For $f \in E^{\prime}$, let $J_{f}$ denote the intersection of $J$ with the corresponding copy of $H$ in $G_{-}^{H}$ (the graph $J_{f}$ may contain isolated vertices). Furthermore, let $I_{0}$ denote the intersection of $J$ with $G_{-}^{\prime}$, and set $V_{0}:=V\left(I_{0}\right)=V(J) \cap V\left(G_{-}^{\prime}\right), E_{0}:=$ $E\left(I_{0}\right)=E(J) \cap E\left(G_{-}^{\prime}\right)$. Observe that the assumption that $J$ is an induced subgraph of $G_{-}^{H}$ implies that also $I_{0}$ is an induced subgraph of $G_{-}^{\prime}$. Furthermore, due to our assumption that $J$ contains the two vertices of $g$, also $I_{0}$ contains the two vertices of $g$.

Note that

$$
e(J)=\sum_{\substack{f \in E^{\prime} \\ v\left(J_{f}\right) \geqslant 2}} e\left(J_{f}\right)
$$

and

$$
\begin{aligned}
v(J) & \geqslant v\left(I_{0}\right)+\sum_{f \in E^{\prime}}\left(v\left(J_{f}\right)-\left|f \cap V_{0}\right|\right)-L\left(G_{-}^{H}\right) \\
& \geqslant v\left(I_{0}\right)+\sum_{\substack{f \in E^{\prime} \\
v\left(J_{f}\right) \geqslant 2}}\left(v\left(J_{f}\right)-\left|f \cap V_{0}\right|\right)-L\left(G_{-}^{H}\right),
\end{aligned}
$$

where the first inequality is due to the fact that the big sum overcounts the actual number of vertices of $J$ by at most $L\left(G_{-}^{H}\right)$ (i.e., $J \subseteq G_{-}^{H}$ 'loses' at most as many vertices as $G_{-}^{H}$ because of vertex-overlapping copies of $H$ ). 
Combining (9) and (10) yields that

$$
\begin{aligned}
& v(J)-\frac{e(J)}{m_{2}(G, H)} \geqslant v\left(I_{0}\right)+\sum_{\substack{f \in E^{\prime} \\
v\left(J_{f}\right) \geqslant 2}} T\left(v\left(J_{f}\right)-\left|f \cap V_{0}\right|-\frac{e\left(J_{f}\right)}{m_{2}(G, H)}\right)-L\left(G_{-}^{H}\right) \\
& \qquad \sum_{\substack{f \in E^{\prime} \\
v\left(J_{f}\right) \geqslant 2}}\left(2-\left|f \cap V_{0}\right|-\frac{1}{m_{2}(G)}\right)-L\left(I_{-}^{H}\right) \\
&=v\left(I_{0}\right)-\frac{e\left(I_{0}\right)}{m_{2}(G)}+\sum_{\substack{f \in E^{\prime} \backslash E_{0} \\
v\left(J_{f}\right) \geqslant 2 \\
\left|f \cap V_{0}\right| \leqslant 1}}\left(2-\left|f \cap V_{0}\right|-\frac{1}{m_{2}(G)}\right)-L\left(G_{-}^{H}\right),
\end{aligned}
$$

where for the equality we used that the edges $f \in E^{\prime}=E\left(G_{-}^{\prime}\right)$ with $\left|f \cap V_{0}\right|=2$ are exactly the edges in $E_{0}=E\left(I_{0}\right)$ due to the fact that $I_{0}$ is an induced subgraph of $G_{-}^{\prime}$. Using that $m_{2}(G) \geqslant 1$, we may omit the remaining sum, and observing that adding the edge $g$ to $I_{0}$ yields a graph $I_{0}^{+}$that is isomorphic to a subgraph of $G$, we obtain further

$$
\begin{aligned}
v(J)-\frac{e(J)}{m_{2}(G, H)} & \geqslant v\left(I_{0}^{+}\right)-\frac{e\left(I_{0}^{+}\right)-1}{m_{2}(G)}-L\left(G_{-}^{H}\right) \\
& \stackrel{(5)}{\geqslant} 2-L\left(G_{-}^{H}\right),
\end{aligned}
$$

concluding the proof of Lemma 10.

2.3. The parameters $m^{*}(H)$ and $x^{*}(H)$. In this section we introduce two graph parameters $m^{*}(H)$ and $x^{*}(H)$ that will play in important role in our proof. The parameter $m^{*}(H)$ is a convenient quantity to capture the concept of $H$ being 'its own least frequent subgraph' that many authors have used before (see Remark 12 below). The parameter $x^{*}(H)$ is a rescaled version of $m^{*}(H)$ that is tailored to the specifics of the problem studied in this paper.

Definition 11. For any graph $H$ with $v_{H} \geqslant 3$, let

$$
m^{*}(H):=\min _{\substack{J \subseteq H: \\ 2 \leqslant v_{J}<v_{H}}} \frac{e_{H}-e_{J}}{v_{H}-v_{J}}
$$

and, if $H$ is nonempty,

$$
x^{*}(H):=\frac{m^{*}(H)}{e_{H}-m^{*}(H)\left(v_{H}-2\right)} .
$$

Note that for any graph $H$, the parameter $m^{*}(H)$ is nonnegative, and that $m^{*}(H)=0$ if and only if $H$ has an isolated vertex. It follows from (11) that for any nonempty graph $H$ with $v_{H} \geqslant 3$ the parameter $x^{*}(H)$ as defined in (12) is well-defined and positive. Note 
that solving (12) for $m^{*}(H)$ yields

$$
m^{*}(H)=\frac{e_{H}}{v_{H}-2+1 / x^{*}(H)},
$$

which connects $m^{*}(H)$ to $d_{2}(G, H)$ as defined in (3). More specifically, the point here is that comparing $m^{*}(H)$ to $d_{2}(G, H)$ can be formulated equivalently as comparing $x^{*}(H)$ to $m_{2}(G)$.

Remark 12. It follows from the definition of $m^{*}(H)$ in (11) that

$$
v_{J}-\frac{e_{J}}{m^{*}(H)} \geqslant v_{H}-\frac{e_{H}}{m^{*}(H)},
$$

for all subgraphs $J \subseteq H$ with $v_{J} \geqslant 2$. Thus for $p \leqslant n^{-1 / m^{*}(H)}$ we have $n^{v_{J}} p^{e_{J}} \geqslant n^{v_{H}} p^{e_{H}}$ for all such $J$, which means that the expected number of copies of $H$ in $G_{n, p}$ does not exceed the expected number of copies of any subgraph $J \subseteq H$ with $v_{J} \geqslant 2$ by more than a constant factor.

In some sense, both $m^{*}(H)$ and $x^{*}(H)$ measure 'how balanced' $H$ is. Below we will prove some general results that make this precise. These will in particular imply the following lemma, which restates the hypothesis of Theorem 4 in two alternative forms that are more convenient for us.

Lemma 13. For any two nonempty graphs $G$ and $H$ with $v_{H} \geqslant 3$, the following statements are equivalent.

(i) $H$ is strictly balanced w.r.t. $d_{2}(G, \cdot)$,

(ii) $m_{2}(G, H)<m^{*}(H)$,

(iii) $m_{2}(G)<x^{*}(H)$.

Lemma 13 will be proved in Section 2.3.2 below.

2.3.1. The parameter $m^{*}(H)$ and general density measures. For arbitrary (possibly negative) values $a \leqslant 1$ and $b<2$, we define for any graph $H$ the density measure

$$
d_{a, b}(H):= \begin{cases}\frac{e_{H}-a}{v_{H}-b} & \text { if } e_{H} \geqslant 1 \\ 0 & \text { otherwise }\end{cases}
$$

and set

$$
m_{a, b}(H):=\max _{J \subseteq H} d_{a, b}(J) .
$$

As usual we say that $H$ is balanced w.r.t. $d_{a, b}$ if $m_{a, b}(H)=d_{a, b}(H)$, and strictly balanced w.r.t. $d_{a, b}$ if in addition $m_{a, b}(H)>d_{a, b}(J)$ for all proper subgraphs $J \subsetneq H$. 
Lemma 14. Let $d_{a, b}$ be a density measure as in (14). A graph $H$ with $v_{H} \geqslant 3$ is balanced w.r.t. $d_{a, b}$ if and only if $m^{*}(H) \geqslant d_{a, b}(H)$ (or, equivalently, if and only if $m^{*}(H) \geqslant$ $\left.m_{a, b}(H)\right)$. Similarly, a graph $H$ with $v_{H} \geqslant 3$ is strictly balanced w.r.t. $d_{a, b}$ if and only if $m^{*}(H)>d_{a, b}(H)$ (or, equivalently, if and only if $m^{*}(H)>m_{a, b}(H)$ ).

For the proof we use the following elementary observation, which we state separately for further reference.

Fact 15. For $a, c \in \mathbb{R}$ and $b>d>0$, we have

$$
\frac{c}{d} \leqslant \frac{a}{b} \Longleftrightarrow \quad \frac{a-c}{b-d} \geqslant \frac{a}{b}
$$

and, similarly,

$$
\frac{c}{d}<\frac{a}{b} \Longleftrightarrow \frac{a-c}{b-d}>\frac{a}{b} .
$$

Proof of Lemma 14. Observe that $H$ is balanced w.r.t. $d_{a, b}$ if and only if for all subgraphs $J \subseteq H$ with $v_{J} \geqslant 2$ we have

$$
\frac{e_{J}-a}{v_{J}-b} \leqslant \frac{e_{H}-a}{v_{H}-b}
$$

(note that this condition is always satisfied for graphs $J$ with $v_{J} \geqslant 2$ and $e_{J}=0$ ). By Fact 15 , this is equivalent to the requirement that

$$
\frac{e_{H}-e_{J}}{v_{H}-v_{J}}=\frac{\left(e_{H}-a\right)-\left(e_{J}-a\right)}{\left(v_{H}-b\right)-\left(v_{J}-b\right)} \stackrel{\text { Fact }}{\geqslant} \stackrel{15}{\frac{e_{H}-a}{v_{H}-b}}
$$

for all subgraphs $J \subseteq H$ with $2 \leqslant v_{J}<v_{H}$, i.e., to $m^{*}(H) \geqslant d_{a, b}(H)$.

The statement for 'strictly balanced' follows analogously using the second statement of Fact 15 .

2.3.2. The parameter $x^{*}(H)$ and the asymmetric 2-density. As a consequence of Lemma 14 we obtain the next lemma, which is specifically concerned with the asymmetric 2-density.

Lemma 16. Let $G$ be a nonempty graph. A nonempty graph $H$ with $v_{H} \geqslant 3$ is balanced w.r.t. $d_{2}(G, \cdot)$ if and only if $m_{2}(G) \leqslant x^{*}(H)$. Similarly, a nonempty graph $H$ with $v_{H} \geqslant 3$ is strictly balanced w.r.t. $d_{2}(G, \cdot)$ if and only if $m_{2}(G)<x^{*}(H)$.

Proof. For any $x>0$ and any graph $H$ with $v_{H} \geqslant 2$, let

$$
d_{2}(x, H):= \begin{cases}\frac{e_{H}}{v_{H}-2+1 / x} & \text { if } e_{H} \geqslant 1 \\ 0 & \text { otherwise, }\end{cases}
$$

and set

$$
m_{2}(x, H):=\max _{J \subseteq H} d_{2}(x, J) .
$$


Note that $d_{2}\left(m_{2}(G), H\right)$ as defined in (15) coincides with $d_{2}(G, H)$ as defined in (3), and that, according to (13), for any nonempty graph $H$ with $v_{H} \geqslant 3$ we have

$$
m^{*}(H)=d_{2}\left(x^{*}(H), H\right)
$$

With Lemma 14 we obtain that $H$ is balanced w.r.t. $d_{2}(G, \cdot)$ if and only if

$$
d_{2}\left(x^{*}(H), H\right) \stackrel{(16)}{=} m^{*}(H) \stackrel{\text { L. } 14}{\geqslant} d_{2}(G, H)=d_{2}\left(m_{2}(G), H\right) .
$$

Since $d_{2}(x, H)$ is monotone increasing in $x$, this is equivalent to $m_{2}(G) \leqslant x^{*}(H)$, as claimed.

The statement for 'strictly balanced' follows analogously using the second statement of Lemma 14.

Proof of Lemma 13. The equivalence of $(i)$ and $(i i)$ follows from Lemma 14, observing that, for any fixed nonempty graph $G$, the parameter $d_{2}(G, H)$ defined in (3) is a density measure as in (14) (with $a=0$ and $\left.b=2-1 / m_{2}(G)\right)$.

The equivalence of $(i)$ and $(i i i)$ is stated in Lemma 16.

2.4. Other preliminaries. As already mentioned, we will make crucial use of the Harris inequality [9] (which also arises as a special case of the FKG inequality [5] and various other related inequalities).

Throughout, we will assume that the random graph $G_{n, p}$ is generated on the vertex set $[n]=\{1, \ldots, n\}$. For the purposes of this paper, a graph property is a family of labelled graphs on the vertex set $[n]$ (which is not necessarily closed under isomorphism), where $n$ will be clear from the context. We say that a graph property $\mathcal{A}$ is decreasing if for any two graphs $G$ and $H$ on vertex set $[n]$ the following holds: if $G \in \mathcal{A}$ and $H \subseteq G$, we also have $H \in \mathcal{A}$. Similarly, we say that a graph property $\mathcal{A}$ is increasing if for any two graphs $G$ and $H$ on vertex set $[n]$ the following holds: if $G \in \mathcal{A}$ and $H \supseteq G$, we also have $H \in \mathcal{A}$. Note that the complement of a decreasing property is increasing, and vice versa.

Theorem 17 (Harris [9]). For any two decreasing (increasing) graph properties $\mathcal{A}$ and $\mathcal{B}$ and any $n \in \mathbb{N}$ and $0 \leqslant p \leqslant 1$, we have

$$
\mathbb{P}\left(G_{n, p} \in \mathcal{A} \cap \mathcal{B}\right) \geqslant \mathbb{P}\left(G_{n, p} \in \mathcal{A}\right) \mathbb{P}\left(G_{n, p} \in \mathcal{B}\right)
$$

or, equivalently if $\mathbb{P}\left(G_{n, p} \in \mathcal{B}\right)>0$,

$$
\mathbb{P}\left(G_{n, p} \in \mathcal{A} \mid G_{n, p} \in \mathcal{B}\right) \geqslant \mathbb{P}\left(G_{n, p} \in \mathcal{A}\right)
$$


Clearly, it follows from Theorem 17 that, for the binomial random graph $G_{n, p}$ the probability of any decreasing (respectively, increasing) event $\mathcal{A}$ does not decrease if we condition on another decreasing (respectively, increasing) event $\mathcal{B}$.

Janson's inequality is a very useful tool in probabilistic combinatorics. In many cases, it yields an exponential bound on lower tails where the second moment method only gives a considerably weaker bound. Here we formulate a version tailored to random graphs.

Theorem 18 (Janson [10]). Consider a family $\mathcal{H}=\left\{H_{i} \mid i \in I\right\}$ of subgraphs of the complete graph on the vertex set $[n]$. For each $H_{i} \in \mathcal{H}$, let $X_{i}$ denote the indicator random variable for the event $H_{i} \subseteq G_{n, p}$, and, for each ordered pair $\left(H_{i}, H_{j}\right) \in \mathcal{H} \times \mathcal{H}$ with $i \neq j$, write $H_{i} \sim H_{j}$ if $H_{i}$ and $H_{j}$ are not edge-disjoint. Let

$$
\begin{aligned}
X & =\sum_{H_{i} \in \mathcal{H}} X_{i}, \\
\mu & =\mathbb{E}[X]=\sum_{H_{i} \in \mathcal{H}} p^{e\left(H_{i}\right)}, \\
\Delta & =\sum_{\substack{\left(H_{i}, H_{j}\right) \in \mathcal{H} \times \mathcal{H} \\
H_{i} \sim H_{j}}} \mathbb{E}\left[X_{i} X_{j}\right]=\sum_{\substack{\left(H_{i}, H_{j}\right) \in \mathcal{H} \times \mathcal{H} \\
H_{i} \sim H_{j}}} p^{e\left(H_{i}\right)+e\left(H_{j}\right)-e\left(H_{i} \cap H_{j}\right)} .
\end{aligned}
$$

Then for all $0 \leqslant \delta \leqslant 1$ we have

$$
\mathbb{P}(X \leqslant(1-\delta) \mu) \leqslant \mathrm{e}^{-\frac{\delta^{2} \mu^{2}}{2(\mu+\Delta)}} .
$$

Often Janson's inequality is applied with $\mathcal{H}$ being the family of all copies of some given fixed graph $H$ in the complete graph $K_{n}$. The concept of $(\varrho, d)$-denseness will allow us to derive very similar results when applying Janson's inequality with $\mathcal{H}$ being the family of all copies of $H$ in a graph $F \subseteq K_{n}$ that is not necessarily complete.

Definition 19. For any $\varrho>0$ and $0<d \leqslant 1$, a graph $F$ on vertex set $[n]$ is said to be $(\varrho, d)$-dense if for every subset $V \subseteq[n]$ with $|V| \geqslant \varrho n$ we have

$$
e(F[V]) \geqslant d\left(\begin{array}{l}
n \\
2
\end{array}\right)
$$

where $F[V]$ denotes the subgraph induced by $F$ on $V$.

We will use the following fact (for a proof see e.g. [21]).

Lemma 20. For all $0<d \leqslant 1$ and $\ell \geqslant 1$, there exist positive constants $\varrho, n_{0}$ and $c_{0}$ such that every $(\varrho, d)$-dense graph on $n \geqslant n_{0}$ vertices contains at least $c_{0} n^{\ell}$ complete subgraphs $K_{\ell}$. 
2.5. Edge-disjoint copies. The tools and definitions presented in the previous section come together in the following technical lemma, which states that under the appropriate assumptions, a random subgraph of a $(\varrho, d)$-dense graph contains a large family of pairwise edge-disjoint copies of a given graph $H$. The key idea of applying Turán's Theorem to a suitably defined auxiliary graph is due to Kreuter [17]. We will use Turán's Theorem in the following form (see e.g. [1, p. 282]).

Theorem 21 (Turán). Let $G$ be a graph. Then $G$ has an independent set of size at least

$$
\frac{v(G)^{2}}{v(G)+2 e(G)} \text {. }
$$

Lemma 22. Let $H$ be a nonempty graph with $v_{H} \geqslant 3$. For any $0<d \leqslant 1$, there exist positive constants $\varrho, n_{0}$ and $b$ such that for $n \geqslant n_{0}$ and $p \leqslant n^{-1 / m^{*}(H)}$ the following holds: If

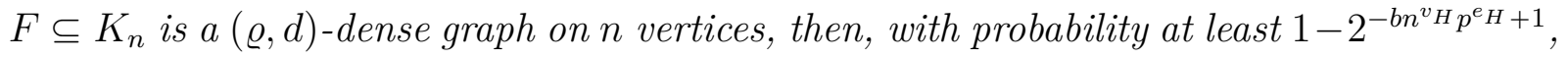
the graph $F \cap G_{n, p}$ contains a family of at least $b n^{v_{H}} p^{e_{H}}$ pairwise edge-disjoint copies of $H$.

Note that for $p=o\left(n^{-1 / m^{*}(H)}\right)$ we have $n^{v_{H}} p^{e_{H}}=o\left(n^{2} p\right)$ (recall Remark 12), so the error probability in Lemma 22 is not as high as it may look like at first glance.

Proof of Lemma 22. Let

$$
\varrho:=\varrho\left(v_{H}, d\right), \quad n_{0}:=n_{0}\left(v_{H}, d\right), \quad c_{0}:=c_{0}\left(v_{H}, d\right) \leqslant 1
$$

denote the constants obtained by applying Lemma 20 with $\ell:=v_{H}$ and $d$. We shall prove Lemma 22 for $\varrho$ and $n_{0}$ as defined in (17) and

$$
b:=\frac{c_{0}^{2}}{16^{v_{H}^{2}+1}} .
$$

Let $F$ be a $(\varrho, d)$-dense graph on $n \geqslant n_{0}$ vertices be given, and set

$$
\mathcal{A}:=\left\{\begin{array}{l|l}
K \subseteq K_{n} & \begin{array}{l}
F \cap K \text { contains a family of at least } b n^{v_{H}} p^{e_{H}} \text { pair- } \\
\text { wise edge-disjoint copies of } H
\end{array}
\end{array}\right\} .
$$

Note that $\mathcal{A}$ is increasing. Our goal is to bound $\mathbb{P}\left(G_{n, p} \in \neg \mathcal{A}\right)$ from above.

Denote by $\mathcal{H}$ the family of all copies of $H$ in $F$. By our choice of constants in (17), the assumption that $F$ is $(\varrho, d)$-dense yields with Lemma 20 that there are at least $c_{0} n^{v_{H}}$ complete graphs of order $v_{H}$ in $F$. In particular, we have

$$
|\mathcal{H}| \geqslant c_{0} n^{v_{H}}
$$

We will apply Janson's inequality (Theorem 18) to the family $\mathcal{H}$. For any graph $K \subseteq K_{n}$, we let $\mathcal{H}(K) \subseteq \mathcal{H}$ denote the family of all copies of $H$ in $F \cap K$. We obtain for $\mu$ as defined in Theorem 18 that

$$
\mu=\mathbb{E}\left[\left|\mathcal{H}\left(G_{n, p}\right)\right|\right]=|\mathcal{H}| \cdot p^{e_{H}} \stackrel{(20)}{\geqslant} c_{0} n^{v_{H}} p^{e_{H}}
$$


Let $\mathcal{S}$ be the family of all pairwise nonisomorphic graphs that are unions of two copies of $H$ that intersect in at least one edge. For a fixed graph $S \in \mathcal{S}$, let $J$ denote the intersection of the two copies of $H$. Owing to the assumption that $p \leqslant n^{-1 / m^{*}(H)}$, we obtain for any nonempty subgraph $J \subseteq H$ that

$$
n^{v_{H}-v_{J}} p^{e_{H}-e_{J}} \leqslant n^{v_{H}-v_{J}-\left(e_{H}-e_{J}\right) / m^{*}(H)} \stackrel{(11)}{\leqslant} 1
$$

which implies that for any $S \in \mathcal{S}$ we have

$$
n^{v_{S}} p^{e_{S}}=n^{2 v_{H}-v_{J}} p^{2 e_{H}-e_{J}} \stackrel{(22)}{\lessgtr} n^{v_{H}} p^{e_{H}} .
$$

As there are at most $n^{v_{S}}$ copies of $S$ in $F$, and since each such copy corresponds to at $\operatorname{most}\left(\left(v_{S}\right)_{v_{H}}\right)^{2} \leqslant\left(2 v_{H}\right)^{2 v_{H}} \leqslant 4^{v_{H}^{2}}$ pairs $\left(H_{i}, H_{j}\right) \in \mathcal{H} \times \mathcal{H}, i \neq j$ with $H_{i} \cup H_{j} \cong S$, we obtain for $\Delta$ as defined in Theorem 18 that

$$
\begin{aligned}
\Delta & =\sum_{\substack { S \in \mathcal{S} \\
\begin{subarray}{c}{\left(H_{i}, H_{j}\right) \in \mathcal{H} \times \mathcal{H} \\
H_{i} \cup H_{j} \cong S{ S \in \mathcal { S } \\
\begin{subarray} { c } { ( H _ { i } , H _ { j } ) \in \mathcal { H } \times \mathcal { H } \\
H _ { i } \cup H _ { j } \cong S } }\end{subarray}} p^{e_{S}} \\
& \leqslant 4^{v_{H}^{2}} \sum_{S \in \mathcal{S}} n^{v_{S}} p^{e_{S}} \\
& \stackrel{(23)}{\leqslant} 16^{v_{H}^{2}} n^{v_{H}} p^{e_{H}},
\end{aligned}
$$

where in the last step we bounded $|\mathcal{S}|$ by the number of graphs on at most $2 v_{H}$ vertices, which in turn is bounded by $\sum_{i=2}^{2 v_{H}} 2^{\left(\begin{array}{c}i \\ 2\end{array}\right)} \leqslant 2^{\left(\begin{array}{c}2 v_{H} \\ 2\end{array}\right)+1} \leqslant 2^{2 v_{H}^{2}}$.

Consider now the property

$$
\mathcal{E}:=\left\{K \subseteq K_{n}|| \mathcal{H}(K) \mid \geqslant \mu / 2\right\}
$$

By Janson's inequality (Theorem 18) we have

$$
\begin{aligned}
& \mathbb{P}\left(G_{n, p} \in \neg \mathcal{E}\right) \leqslant \exp \left(-\frac{\mu^{2}}{8(\mu+\Delta)}\right) \leqslant \exp \left(-\frac{1}{16} \cdot \min \left\{\mu, \frac{\mu^{2}}{\Delta}\right\}\right) \\
& \stackrel{(21),(24)}{\leqslant} \exp \left(-\frac{1}{16} \min \left\{c_{0}, \frac{c_{0}^{2}}{16^{v_{H}^{2}}}\right\} n^{v_{H}} p^{e_{H}}\right) \stackrel{(18)}{\leqslant} \exp \left(-b n^{v_{H}} p^{e_{H}}\right),
\end{aligned}
$$

where in the second to last step we also used that $c_{0} \leqslant 1$ (see $(17)$ ).

For a given graph $K \subseteq K_{n}$, consider the auxiliary graph $\widetilde{G}=\widetilde{G}(K)$ on the vertex set $V(\widetilde{G})=\mathcal{H}(K)$, in which two vertices are connected by an edge if and only if those two copies of $H$ are not edge-disjoint.

Note that

$$
\mathbb{E}\left[e\left(\widetilde{G}\left(G_{n, p}\right)\right)\right]=\Delta / 2
$$


for $\Delta$ as in (24) (the factor $1 / 2$ is due to the fact that the sum in (24) is over ordered pairs). Thus for the property

$$
\mathcal{D}:=\left\{K \subseteq K_{n} \mid e(\widetilde{G}(K)) \leqslant \Delta\right\}
$$

we obtain with Markov's inequality that

$$
\mathbb{P}\left(G_{n, p} \in \mathcal{D}\right) \geqslant 1 / 2
$$

By definition of the auxiliary graph $\widetilde{G}=\widetilde{G}(K)$, any independent set in $\widetilde{G}$ corresponds to a family $\widetilde{\mathcal{H}} \subseteq \mathcal{H}(K)$ of pairwise edge-disjoint copies of $H$ in $F \cap K$. Thus our definitions of $\mathcal{D}$ and $\mathcal{E}$ imply with Turán's Theorem (Theorem 21) that any graph $K \in \mathcal{D} \cap \mathcal{E}$ contains a subfamily $\tilde{\mathcal{H}} \subseteq \mathcal{H}(K)$ of pairwise edge-disjoint copies of $H$ of size at least

$$
\begin{aligned}
|\widetilde{\mathcal{H}}| \stackrel{\text { Thm. } 21}{\geqslant} \frac{v(\widetilde{G})^{2}}{v(\widetilde{G})+2 e(\widetilde{G})} \geqslant \frac{1}{2} \cdot \min \left\{v(\widetilde{G}), \frac{v(\widetilde{G})^{2}}{2 e(\widetilde{G})}\right\} & \stackrel{(25),(27)}{\geqslant} \frac{1}{16} \min \left\{4 \mu, \frac{\mu^{2}}{\Delta}\right\} \geqslant b n^{v_{H}} p^{e_{H}}
\end{aligned}
$$

where the last inequality follows analogously to (26). In other words, we have just shown that

$$
\mathcal{D} \cap \mathcal{E} \subseteq \mathcal{A}
$$

or, equivalently,

$$
\neg \mathcal{A} \cap \mathcal{D} \subseteq \neg \mathcal{E}
$$

Since $\neg \mathcal{A}$ and $\mathcal{D}$ are both decreasing, we obtain with the Harris inequality (Theorem 17) that

$$
\begin{aligned}
\mathbb{P}\left(G_{n, p} \in \neg \mathcal{A}\right) & \stackrel{\text { Thm. } 17}{\lessgtr} \mathbb{P}\left(G_{n, p} \in \neg \mathcal{A} \mid G_{n, p} \in \mathcal{D}\right) \\
& \stackrel{(28)}{\leqslant} 2 \mathbb{P}\left(G_{n, p} \in \neg \mathcal{A} \cap \mathcal{D}\right) \stackrel{(30)}{\leqslant} 2 \mathbb{P}\left(G_{n, p} \in \neg \mathcal{E}\right) \\
& \stackrel{(26)}{\leqslant} 2 \exp \left(-b n^{v_{H}} p^{e_{H}}\right) \leqslant 2^{1-b n^{v_{H}} p^{e_{H}}},
\end{aligned}
$$

as claimed.

\section{§3. Proof of Theorem 4}

As already mentioned, our proof of Theorem 4 proceeds by induction on $e(G)$, whereas $H$ is considered fixed. In order for this induction to work, we will prove the following stronger statement. Recall that we introduced the set of $H$-edges $E_{H}\left(G_{n, p}\right)$ and the notion of $H$ covered copies in Definition 8. 
Lemma 23 (Main lemma). Let $H$ be a nonempty graph with $v_{H} \geqslant 3$. For any nonempty graph $G$ satisfying $m_{2}(G)<x^{*}(H)$ there exist positive constants $a, b, C$, and $n_{0}$ such that for $n \geqslant n_{0}$ and

$$
C n^{-1 / m_{2}(G, H)} \leqslant p \leqslant n^{-1 / m^{*}(H)},
$$

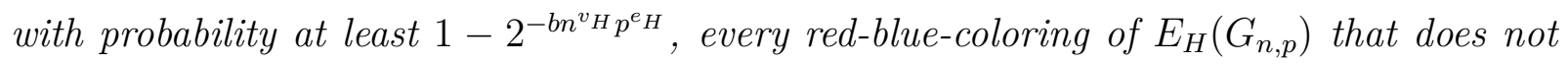
contain a blue copy of $H$ contains at least $a n^{v_{G}}\left(n^{v_{H}-2} p^{e_{H}}\right)^{e_{G}}$ many $H$-covered red copies of $G$.

Note that, because of (8), the number of $H$-covered red copies of $G$ guaranteed by Lemma 23 is of the same order of magnitude as the expected number of copies of graphs from $\mathcal{F}(G, H)$ (as defined in Definition 9) in $G_{n, p}$.

Remark 24. The statement of Lemma 23 is void if $H$ is not strictly balanced w.r.t. $d_{2}(G, \cdot)$ (recall Lemma 13). On the other hand, for $H$ strictly balanced w.r.t. $d_{2}(G, \cdot)$, there is $p$ as in (31), and - crucially for our proof of Lemma 23 - we may apply Lemma 22 for such $p$.

Remark 25. For the two-color case studied here, it would be sufficient to prove the statement of Lemma 23 with an error probability of $2^{-\Theta(n)}$ instead of $2^{-\Theta\left(n^{v} H p^{e} H\right)}$ (see (72) below). However, our arguments yield the latter for free, and this is also what would be needed to extend our inductive approach to more than two colors.

Lemma 23 implies Theorem 4 as follows.

Proof of Theorem 4. Owing to Lemma 13, $G$ and $H$ as in Theorem 4 satisfy the hypothesis of Lemma 23. We will prove Theorem 4 for the constant $C=C(G, H)$ guaranteed by Lemma 23.

By monotonicity it suffices to prove the theorem for $p=p(n):=C n^{-1 / m_{2}(G, H)}$. Again due to Lemma 13, this is smaller than $n^{-1 / m^{*}(H)}$ for $n$ large enough, and thus Lemma 23 is applicable for this $p=p(n)$. Clearly, if the event in Lemma 23 holds then we have in particular that $G_{n, p} \rightarrow(G, H)$. Furthermore, due to (6) and the assumption that $G$ is not a matching, $n^{v_{H}} p^{e_{H}}$ is a growing function of $n$. Hence the probability stated in Lemma 23 is indeed $1-o(1)$, and Theorem 4 is proved.

3.1. Proof of Lemma 23. It remains to prove Lemma 23, which we will do in the remainder of this section. Our main proof hinges on two fairly involved statements (Claim 26 and Claim 27 below), whose proofs are deferred to Section 3.2 and Section 3.3, respectively.

As already mentioned, we proceed by induction on $e(G)$. Our induction base is the case where $G$ is a matching. 
Proof of Lemma 23: Induction base $-G$ is a matching. W.l.o.g. we may assume that $G$ contains no isolated vertices, i.e., that $v_{G}=2 e_{G}$. Let

$$
\varrho:=\varrho(H, 1), \quad n^{\prime}:=n_{0}(H, 1), \quad b^{\prime}:=b(H, 1)
$$

denote the constants obtained by applying Lemma 22 for $H$ and $d:=1$. We shall prove Lemma 23 for

$$
\begin{aligned}
a=a(G, H) & :=\left(\frac{b^{\prime}}{\left(2 e_{G}\right)^{v_{H}}}\right)^{e_{G}}, \\
b=b(G, H) & :=\frac{b^{\prime}}{\left(\log _{2} e_{G}+2\right) \cdot\left(2 e_{G}\right)^{v_{H}}}, \\
C=C(G, H) & :=b^{-1 / e_{H}}, \\
n_{0}=n_{0}(G, H) & :=\left(2 e_{G}\right) \cdot n^{\prime} .
\end{aligned}
$$

Note that for any $n \geqslant 1$ we have

$$
1=n^{2-1 / m_{2}(G) \stackrel{(6),(35)}{\leqslant}} b C^{e_{H}} n^{v_{H}-e_{H} / m_{2}(G, H)} \stackrel{(31)}{\leqslant} b n^{v_{H}} p^{e_{H}} .
$$

Fix $e_{G}$ pairwise disjoint sets $V_{1}, \ldots, V_{e_{G}} \subseteq[n]$ of size

$$
\tilde{n}:=\left\lfloor n / e_{G}\right\rfloor \geqslant n /\left(2 e_{G}\right)
$$

each, and note that the graphs $G_{n, p}\left[V_{i}\right]$ induced by $G_{n, p}$ on these sets behave like independent random graphs $G_{\widetilde{n}, p}$, where $\tilde{n} \geqslant n^{\prime}$ due to our choice of $n_{0}$ in (36).

Due to our choice of constants in (32) and observing that the complete graph $K_{\tilde{n}}$ is $(\varrho, 1)$ dense, we obtain with Lemma 22 and the union bound that for $n \geqslant n_{0}$, with probability at least

$$
\begin{aligned}
1-e_{G} \cdot 2^{1-b^{\prime} \tilde{n}^{v} H p^{e} H} & \geqslant 1-2^{\log _{2} e_{G}+1-\left(b^{\prime} /\left(2 e_{G}\right)^{v} H\right) n^{v} H p^{e} H} \\
& \stackrel{(34)}{=} 1-2^{\log _{2} e_{G}+1-\left(\log _{2} e_{G}+2\right) b n^{v} H p^{e} H} \stackrel{(37)}{\geqslant} 1-2^{-b n^{v} H p^{e} H},
\end{aligned}
$$

each of the graphs $G_{n, p}\left[V_{i}\right]$ contains a family of at least $b^{\prime} \tilde{n}^{v_{H}} p^{e_{H}}$ pairwise edge-disjoint copies of $H$. To avoid creating a blue copy of $H$, one edge from each of these copies needs to be colored red. Thus in every red-blue-coloring of $E_{H}\left(G_{n, p}\right)$ there is either a blue copy of $H$ or we can obtain at least

$$
\left(b^{\prime} \tilde{n}^{v_{H}} p^{e_{H}}\right)^{e_{G}} \stackrel{(33),(38)}{\geqslant} a n^{2 e_{G}}\left(n^{v_{H}-2} p^{e_{H}}\right)^{e_{G}}=a n^{v_{G}}\left(n^{v_{H}-2} p^{e_{H}}\right)^{e_{G}}
$$

many red matchings by picking exactly one $H$-covered red edge from each of the graphs $G_{n, p}\left[V_{i}\right], 1 \leqslant i \leqslant e_{G}$. By definition, these red matchings are $H$-covered red copies of $G$. 
Before giving the proof of the induction step, let us give an informal outline of the key proof ideas. As already mentioned, our approach can be seen as a refinement of the proof for the symmetric case given by Rödl and Ruciński in [21]. We will generate $G_{n, p}$ in two rounds, i.e., as the union of two independent binomial random graphs $G_{n, p_{1}}$ and $G_{n, p_{2}}$ on the same vertex set. Let $G_{-}$denote a fixed subgraph of $G$ with $e(G)-1$ edges and $v(G)$ vertices. By the induction hypothesis, with high probability every coloring of the $H$-edges of the first round that does not contain a blue copy of $H$ contains 'many' $H$-covered red copies of $G_{-}$. Each of those induces a vertex pair that will complete a red copy of $G$ if it is sampled as an edge of the second round and is colored red. In our argument we will consider vertex pairs that complete not only one, but 'many' red copies of $G_{-}$to copies of $G$. We will call the graph spanned by these edges the base graph $\Gamma(h)$ of a given coloring $h$ of $E_{H}\left(G_{n, p_{1}}\right)$, the $H$-edges of the first round. Our main goal when analyzing the first round is to show that, with suitably high probability, the base graph $\Gamma(h)$ is $(\varrho, d)$ dense for every coloring $h$ of $E_{H}\left(G_{n, p_{1}}\right)$ (for appropriately chosen parameters $\varrho$ and $d$ ). Once this is shown, we may apply Lemma 22 to find 'many' pairwise edge-disjoint copies of $H$ in $\Gamma(h) \cap G_{n, p_{2}}$, the random subgraph of $\Gamma(h)$ spanned by the edges of the second round. In order to avoid creating a blue copy of $H$, one edge from each such copy needs to be colored red, which by definition of the base graph $\Gamma(h)$ creates 'many' $H$-covered red copies of $G$.

For this approach to work, the arguments of the second round need to work for all possible colorings of the $H$-edges of the first round simultaneously. In order to infer this with the union bound, we need that for a fixed coloring $h$ of the first round, the second round fails with probability exponentially small in the number of $H$-edges. Here it is crucial that we only consider colorings of the $H$-edges of the first round, as the error probability for the second round is not small enough to beat the number of colorings of all edges of the first round!

Proof of Lemma 23: Induction step - $G$ is not a matching. We denote by $G$ - an arbitrary fixed subgraph of $G$ with $e(G)-1$ edges and $v(G)$ vertices. Note that we imposed no balancedness restricion on $G$, and hence both $G$ and $G_{-}$may be disconnected and even contain isolated vertices.

We start by fixing all constants needed in the proof. Throughout the following, by $a\left(G_{-}, H\right)$ etc we denote the constants guaranteed inductively by Lemma 23 .

Let

$$
\ell:=2\left(v_{G}+\left(e_{G}-1\right)\left(v_{H}-2\right)\right)
$$


and set

$$
d:=\frac{a\left(G_{-}, H\right)^{2}}{12 \cdot 2^{\ell^{2}} \cdot \ell^{2 v_{G}}} .
$$

Let

$$
\varrho:=\varrho(H, d), \quad n^{\prime}:=n_{0}(H, d), \quad b^{\prime}:=b(H, d)
$$

denote the constants obtained by applying Lemma 22 for $H$ and $d$. Set

$$
c_{\Gamma}:=\varrho^{\left(v_{G}-2\right)+\left(e_{G}-1\right)\left(v_{H}-2\right)} \cdot a\left(G_{-}, H\right)
$$

and

$$
C_{1}:=\max \left\{C\left(G_{-}, H\right),\left(\frac{3}{c_{\Gamma}}\right)^{\frac{1}{e_{H}\left(e_{G}-1\right)}}\right\} .
$$

Fix $\alpha>0$ small enough such that

$$
\alpha^{e_{H}} \leqslant \frac{b^{\prime}}{16 e_{H}} \quad \text { and } \quad(1-\alpha)^{e_{H}} \geqslant 1 / 2
$$

and set

$$
\begin{aligned}
b_{1} & :=\frac{1}{2} b\left(G_{-}, H\right) \varrho^{v_{H}} \alpha^{e_{H}}, \\
b_{2} & :=b^{\prime} / 4 .
\end{aligned}
$$

We shall prove Lemma 23 for

$$
\begin{aligned}
a=a(G, H) & :=\left(b^{\prime} / 2\right) \cdot c_{\Gamma} \cdot \alpha^{\left(e_{G}-1\right) e_{H}}, \\
b=b(G, H) & :=\frac{1}{2} \min \left\{b_{1}, b_{2} / 2\right\}, \\
C=C(G, H) & :=\max \left\{\frac{C_{1}}{\alpha \cdot \varrho^{1 / m_{2}(G, H)}}, b^{-1 / e_{H}}\right\}, \\
n_{0}=n_{0}(G, H) & :=\max \left\{\frac{n_{0}\left(G_{-}, H\right)}{\varrho}, n^{\prime}\right\} .
\end{aligned}
$$

Let $n \geqslant n_{0}$ and $p$ as in (31) be given, and set

$$
p_{1}:=\alpha p, \quad p_{2}:=\frac{p-p_{1}}{1-p_{1}} .
$$

Note that

$$
(1-\alpha) p \leqslant p_{2} \leqslant p
$$

Throughout the proof we will identify $G_{n, p}$ with the union of two independent random graphs $G_{n, p_{1}}$ and $G_{n, p_{2}}$ on the same vertex set $[n]$. Note that indeed each edge of $K_{n}$ is included in $G_{n, p_{1}} \cup G_{n, p_{2}}$ with probability

$$
1-\left(1-p_{1}\right)\left(1-p_{2}\right) \stackrel{(51)}{=} p
$$

independently. 
As $G$ is not a matching, we have $m_{2}(G) \geqslant 1$, and consequently for any $n \geqslant 1$ that

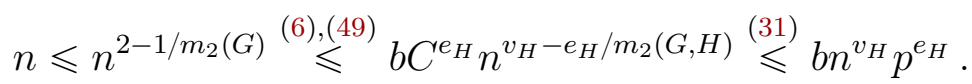

Next we define a number of graph properties to formalize the ideas outlined above. Throughout, $\mathcal{A}, \mathcal{B}, \mathcal{C}$ etc. denote 'good' properties, i.e., properties that are desirable in our proofs.

Let

$$
\mathcal{A}:=\left\{\begin{array}{l|l}
K \subseteq K_{n} & \begin{array}{l}
\text { Every red-blue-coloring of } E_{H}(K) \text { that does not } \\
\text { contain a blue copy of } H \text { contains at least } \\
a n^{v_{G}}\left(n^{v_{H}-2} p^{e_{H}}\right)^{e_{G}} \text { many } H \text {-covered red copies of } \\
G
\end{array}
\end{array}\right\},
$$

and note that $\mathcal{A}$ is an increasing graph property. Our goal is to bound $\mathbb{P}\left(G_{n, p} \in \neg \mathcal{A}\right)$ from above.

For any graph $K \subseteq K_{n}$ (representing a fixed outcome of $G_{n, p_{1}}$ ) and any red-blue coloring $h$ of $E_{H}(K)$, set

$$
\mathcal{A}_{K, h}:=\left\{\begin{array}{l|l}
K^{\prime} \subseteq K_{n} & \begin{array}{l}
\text { Every extension of } h \text { to } E_{H}\left(K \cup K^{\prime}\right) \text { that does } \\
\text { not contain a blue copy of } H \text { contains at least } \\
a n^{v_{G}}\left(n^{v_{H}-2} p^{e_{H}}\right)^{e_{G}} \text { many } H \text {-covered red copies of } \\
G
\end{array}
\end{array}\right\} .
$$

Note that $\mathcal{A}_{K, h}$ is increasing for any fixed $K$ and $h$.

Let

$$
z:=c_{\Gamma} \cdot n^{v_{G}-2}\left(n^{v_{H}-2} p_{1}^{e_{H}}\right)^{e_{G}-1} .
$$

where $c_{\Gamma}$ is defined in (42). For any graph $K \subseteq K_{n}$ (again representing a fixed outcome of $\left.G_{n, p_{1}}\right)$ and any red-blue-coloring $h$ of $E_{H}(K)$, set

$$
\Gamma(K, h):=\left\{\begin{array}{l|l}
e \in\left(\begin{array}{c}
{[n]} \\
2
\end{array}\right) & \begin{array}{l}
e \text { completes at least } z \text { many } H \text {-covered copies of } \\
G_{-} \text {in } E_{H}(K) \text { that are colored red in } h \text { to copies } \\
\text { of } G
\end{array}
\end{array}\right\} .
$$

We will refer to the graph $([n], \Gamma(K, h)) \subseteq K_{n}$ as the base graph determined by the coloring $h$. Further, let

$$
\mathcal{B}:=\left\{\begin{array}{l|l}
K \subseteq K_{n} & \begin{array}{l}
\text { For every red-blue-coloring } h \text { of } E_{H}(K) \text { that does } \\
\text { not contain a blue copy of } H, \text { the base graph } \\
\Gamma(K, h) \text { is }(\varrho, d) \text {-dense }
\end{array}
\end{array},\right.
$$

where $d$ and $\varrho$ are defined in (40) and (41). Note that $\mathcal{B}$ is an increasing graph property. Finally, let

$$
\mathcal{C}:=\left\{K \subseteq K_{n}|| E_{H}(K) \mid \leqslant 2 e_{H} \cdot n^{v_{H}} p_{1}^{e_{H}}\right\}
$$

and note that $\mathcal{C}$ is a decreasing graph property. 
We will prove the following two claims.

Claim 26. We have

$$
\mathbb{P}\left(G_{n, p_{1}} \in \neg \mathcal{B}\right) \leqslant 2^{-b_{1} n^{v} H p^{e} H} .
$$

Claim 27. For every $K \in \mathcal{B}$ and every red-blue-coloring $h$ of $E_{H}(K)$, we have

$$
\mathbb{P}\left(G_{n, p_{2}} \in \neg \mathcal{A}_{K, h}\right) \leqslant 2^{-b_{2} n^{v} H p^{e} H} .
$$

Claim 26 and Claim 27 imply Lemma 23 as follows. Recall that our goal is to bound $\mathbb{P}\left(G_{n, p} \in \neg \mathcal{A}\right)$ from above, and that we generate $G_{n, p}$ as the union of two independent random graphs $G_{n, p_{1}}$ and $G_{n, p_{2}}$.

As the expected number of copies of $H$ in $G_{n, p_{1}}$ is bounded by $n^{v_{H}} p_{1}^{e_{H}}$, Markov's inequality yields for $\mathcal{C}$ defined in (59) that

$$
\mathbb{P}\left(G_{n, p_{1}} \in \mathcal{C}\right) \geqslant 1 / 2 .
$$

For any graph $K^{\prime} \subseteq K_{n}$ (representing a fixed outcome of $G_{n, p_{2}}$ ) we set

$$
\mathcal{A}_{K^{\prime}}:=\left\{K \subseteq K_{n} \mid K \cup K^{\prime} \in \mathcal{A}\right\}
$$

where $\mathcal{A}$ is defined in (54). As $\mathcal{A}$ is increasing, also the property $\mathcal{A}_{K^{\prime}}$ is increasing for any $K^{\prime} \subseteq K_{n}$. Thus its complement is decreasing, and we obtain with the Harris inequality (Theorem 17) that for any $K^{\prime} \subseteq K_{n}$ we have

$$
\mathbb{P}\left(G_{n, p_{1}} \in \neg \mathcal{A}_{K^{\prime}}\right) \stackrel{\text { Thm. }}{\leqslant} \mathbb{P}\left(G_{n, p_{1}} \in \neg \mathcal{A}_{K^{\prime}} \mid G_{n, p_{1}} \in \mathcal{C}\right) \stackrel{(60)}{\leqslant} 2 \mathbb{P}\left(G_{n, p_{1}} \in \neg \mathcal{A}_{K^{\prime}} \cap \mathcal{C}\right) .
$$

Using the independence of $G_{n, p_{1}}$ and $G_{n, p_{2}}$ and the law of total probability, we can infer that

$$
\begin{aligned}
\mathbb{P}\left(G_{n, p} \in \neg \mathcal{A}\right) & =\sum_{K^{\prime} \subseteq K_{n}} \mathbb{P}\left[G_{n, p_{1}} \in \neg \mathcal{A}_{K^{\prime}}\right] \cdot \mathbb{P}\left[G_{n, p_{2}}=K^{\prime}\right] \\
& \stackrel{(61)}{\leqslant} 2\left(\sum_{K^{\prime} \subseteq K_{n}} \mathbb{P}\left[G_{n, p_{1}} \in \neg \mathcal{A}_{K^{\prime}} \cap \mathcal{C}\right] \cdot \mathbb{P}\left[G_{n, p_{2}}=K^{\prime}\right]\right) \\
& =2 \mathbb{P}\left(\left(G_{n, p} \in \neg \mathcal{A}\right) \wedge\left(G_{n, p_{1}} \in \mathcal{C}\right)\right) .
\end{aligned}
$$

Thus it suffices to bound the last probability. Again by the law of total probability, we have

$$
\begin{aligned}
& \mathbb{P}\left(\left(G_{n, p} \in \neg \mathcal{A}\right) \wedge\left(G_{n, p_{1}} \in \mathcal{C}\right)\right) \\
= & \mathbb{P}\left(\left(G_{n, p} \in \neg \mathcal{A}\right) \wedge\left(G_{n, p_{1}} \in \neg \mathcal{B} \cap \mathcal{C}\right)\right) \\
& \quad+\sum_{K \in \mathcal{B} \cap \mathcal{C}} \mathbb{P}\left(G_{n, p} \in \neg \mathcal{A} \mid G_{n, p_{1}}=K\right) \mathbb{P}\left(G_{n, p_{1}}=K\right) \\
\leqslant & \mathbb{P}\left(G_{n, p_{1}} \in \neg \mathcal{B}\right)+\max _{K \in \mathcal{B} \cap \mathcal{C}} \mathbb{P}\left(G_{n, p} \in \neg \mathcal{A} \mid G_{n, p_{1}}=K\right) .
\end{aligned}
$$


Observe that if $G_{n, p_{1}}=K$ we have $G_{n, p} \in \neg \mathcal{A}$ if and only if $G_{n, p_{2}} \in \bigcup_{h} \neg \mathcal{A}_{K, h}$, where the union is over all $2^{\left|E_{H}(K)\right|}$ red-blue colorings $h$ of $E_{H}(K)$. Together with the independence of $G_{n, p_{1}}$ and $G_{n, p_{2}}$ it follows that for any $K \subseteq K_{n}$ we have

$$
\mathbb{P}\left(G_{n, p} \in \neg \mathcal{A} \mid G_{n, p_{1}}=K\right)=\mathbb{P}\left(G_{n, p_{2}} \in \bigcup_{h} \neg \mathcal{A}_{K, h}\right)
$$

If in addition $K$ is in $\mathcal{C}$ as defined in (59), we obtain with

$$
\left|E_{H}(K)\right| \stackrel{(59)}{\leqslant} 2 e_{H} \cdot n^{v_{H}} p_{1}^{e_{H}} \stackrel{(44),(51)}{\leqslant}\left(b^{\prime} / 8\right) n^{v_{H}} p^{e_{H}} \stackrel{(46)}{=}\left(b_{2} / 2\right) n^{v_{H}} p^{e_{H}}
$$

and the union bound that

$$
\mathbb{P}\left(G_{n, p} \in \neg \mathcal{A} \mid G_{n, p_{1}}=K\right) \leqslant 2^{\left(b_{2} / 2\right) n^{v} H p^{e} H} \cdot \max _{h} \mathbb{P}\left(G_{n, p_{2}} \in \neg \mathcal{A}_{K, h}\right),
$$

where the maximum is over all red-blue colorings $h$ of $E_{H}(K)$.

Combining (62), (63), and (64), we obtain that

$$
\begin{aligned}
& \mathbb{P}\left(G_{n, p} \in \neg \mathcal{A}\right) \leqslant 2\left(\mathbb{P}\left(G_{n, p_{1}} \in \neg \mathcal{B}\right)\right.\left.+2^{\left(b_{2} / 2\right) n^{v} H p^{e} H} \max _{K \in \mathcal{B}, h} \mathbb{P}\left(G_{n, p_{2}} \in \neg \mathcal{A}_{K, h}\right)\right) \\
& \stackrel{\text { Cl. } 26, \text { Cl. } 27}{\leqslant} 2\left(2^{-b_{1} n^{v} H p^{e_{H}}}+2^{-\left(b_{2} / 2\right) n^{v} H p^{e} H}\right) \\
& \leqslant 4 \cdot 2^{-\min \left\{b_{1}, b_{2} / 2\right\} n^{v} H p^{e} H} \stackrel{(\underline{48)}}{=} 2^{2-2 b n^{v} H p^{e} H} \leqslant 2^{-b n^{v} H p^{e} H},
\end{aligned}
$$

where in the last step we used that $2 \leqslant b n^{v_{H}} p^{e_{H}}$ due to (53).

It remains to prove Claim 26 and Claim 27.

3.2. Proof of Claim 26. We start with the proof of Claim 26, which concerns the 'probability of failure' of the first round $G_{n, p_{1}}$.

Proof of Claim 26. In order to verify that a graph $F \subseteq K_{n}$ is $(\varrho, d)$-dense, an averaging argument shows that it suffices to check that every set $V \subseteq[n]$ of size

$$
\widetilde{n}:=\lceil\varrho n\rceil
$$

contains at least $d\left(\begin{array}{l}\widetilde{n} \\ 2\end{array}\right)$ edges of $F$ (see [21]).

For any graph $K \subseteq K_{n}$ (representing a fixed outcome of $G_{n, p_{1}}$ ), any red-blue-coloring $h$ of $E_{H}(K)$, and any set $V \subseteq[n],|V|=\tilde{n}$, set

$$
\Gamma(K, h, V):=\left\{e \in\left(\begin{array}{l}
V \\
2
\end{array}\right) \mid \begin{array}{l}
e \text { completes at least } z \text { many } H \text {-covered copies of } \\
G_{-} \text {in } E_{H}(K[V]) \text { that are colored red in } h \text { to } \\
\text { copies of } G
\end{array}\right\},
$$


where $z$ is defined in (56), and define

$$
\mathcal{B}_{V}:=\left\{\begin{array}{l|l}
K \subseteq K_{n} & \begin{array}{l}
\text { For every red-blue-coloring } h \text { of } E_{H}(K) \text { that } \\
\text { does not contain a blue copy of } H, \text { we have } \\
|\Gamma(K, h, V)| \geqslant d \widetilde{n}^{2}
\end{array}
\end{array} .\right.
$$

Note that $\mathcal{B}_{V}$ is increasing.

For a fixed set $V \subseteq[n],|V|=\tilde{n}$, and for any red-blue coloring $h$ of $E_{H}(K)$, let $k_{G_{-}}(K, h, V)$ denote the total number of $H$-covered red copies of $G_{-}$in $E_{H}(K[V])$, and set

$$
\mathcal{A}_{V}:=\left\{\begin{array}{l|l}
K \subseteq K_{n} & \begin{array}{l}
\text { For every red-blue-coloring } h \text { of } E_{H}(K) \text { that does not } \\
\text { contain a blue copy of } H, \text { we have } \\
k_{G_{-}}(K, h, V) \geqslant a\left(G_{-}, H\right) \cdot \widetilde{n}^{v_{G}}\left(\widetilde{n}^{v_{H}-2} p_{1}^{e_{H}}\right)^{e_{G}-1}
\end{array}
\end{array}\right\}
$$

Note that $\mathcal{A}_{V}$ is increasing.

Recall that $H$-covered copies of $G_{-}$are copies of $G_{-}$that are a central copy in a copy of a graph $G_{-}^{H} \in \mathcal{F}\left(G_{-}, H\right)$ as defined in Definition 9. Let $\mathcal{T}$ be the family of all pairwise nonisomorphic graphs $T$ which are unions of two graphs from $\mathcal{F}\left(G_{-}, H\right)$, say $G_{1-}^{H}$ and $G_{2-}^{H}$, such that some vertex pair $g \in\left(\begin{array}{c}V(T) \\ 2\end{array}\right)$ completes both a central copy in $G_{1-}^{H}$ and a central copy in $G_{2-}^{H}$ to a copy of $G$. For any graph $K \subseteq K_{n}$ and any set $V \subseteq[n],|V|=\tilde{n}$, let $k_{\mathcal{T}}(K, V)$ denote the number of copies of graphs from $\mathcal{T}$ in $K[V]$, and set

$$
\mathcal{D}_{V}:=\left\{K \subseteq K_{n} \mid k_{\mathcal{T}}(K, V) \leqslant 2^{\ell^{2}} \widetilde{n}^{2 v_{G}-2}\left(\widetilde{n}^{v_{H}-2} p_{1}^{e_{H}}\right)^{2\left(e_{G}-1\right)}\right\}
$$

Note that $\mathcal{D}_{V}$ is decreasing.

We will show the following three statements.

Fact 28. For every fixed set $V \subseteq[n],|V|=\tilde{n}$, we have $\mathbb{P}\left(G_{n, p_{1}} \in \neg \mathcal{A}_{V}\right) \leqslant 2^{-2 b_{1} n^{v} H p^{e_{H}}}$.

Fact 29. For every fixed set $V \subseteq[n],|V|=\tilde{n}$, we have $\mathbb{P}\left(G_{n, p_{1}} \in \mathcal{D}_{V}\right) \geqslant 1 / 2$.

Fact 30. For every fixed set $V \subseteq[n],|V|=\tilde{n}$, we have $\mathcal{A}_{V} \cap \mathcal{D}_{V} \subseteq \mathcal{B}_{V}$.

With these statements in hand, Claim 26 can be deduced as follows. Note that Fact 30 is equivalent to

$$
\neg \mathcal{B}_{V} \cap \mathcal{D}_{V} \subseteq \neg \mathcal{A}_{V}
$$

Since $\neg \mathcal{B}_{V}$ and $\mathcal{D}_{V}$ are both decreasing, we obtain with the Harris inequality (Theorem 17) that 


$$
\begin{gathered}
\mathbb{P}\left(G_{n, p_{1}} \in \neg \mathcal{B}_{V}\right) \stackrel{\text { Thm. }}{\stackrel{17}{\leqslant}} \mathbb{P}\left(G_{n, p_{1}} \in \neg \mathcal{B}_{V} \mid G_{n, p_{1}} \in \mathcal{D}_{V}\right) \\
\stackrel{\text { Fact } 29}{\lessgtr} 2 \mathbb{P}\left(G_{n, p_{1}} \in \neg \mathcal{B}_{V} \cap \mathcal{D}_{V}\right) \\
\stackrel{\text { Fact } 30}{\leqslant} 2 \mathbb{P}\left(G_{n, p_{1}} \in \neg \mathcal{A}_{V}\right) \\
\stackrel{\text { Fact } 28}{\leqslant} 2^{1-2 b_{1} n^{v_{H} p^{e} H}} .
\end{gathered}
$$

By definition of $\mathcal{B}$ and $\mathcal{B}_{V}$ (see (58) and (67)), we have

$$
\neg \mathcal{B} \subseteq \bigcup_{\substack{V \subseteq[n]: \\|V|=\widetilde{n}}} \neg \mathcal{B}_{V}
$$

Taking the union bound over all sets $V \subseteq[n]$ with $|V|=\tilde{n}$ we obtain

$$
\mathbb{P}\left(G_{n, p_{1}} \in \neg \mathcal{B}\right) \stackrel{(71)}{\leqslant} \sum_{\substack{V \subseteq[n]: \\|V|=\tilde{n}}} \mathbb{P}\left(G_{n, p_{1}} \in \neg \mathcal{B}_{V}\right) \stackrel{(70)}{\leqslant} 2^{n+1-2 b_{1} n^{v} H p^{e} H} \leqslant 2^{-b_{1} n^{v} H p^{e} H}
$$

where in the last step we used that $n+1 \leqslant b_{1} n^{v_{H}} p^{e_{H}}$ due to (53) and (48).

It remains to prove Facts 28, 29, and 30. For all these proofs, note that $G_{n, p_{1}}[V]$ behaves exactly like a binomial random graph $G_{\widetilde{n}, p_{1}}$, and that

$$
p_{1} \stackrel{(31),(51)}{\geqslant} \alpha C n^{-1 / m_{2}(G, H)} \stackrel{(49),(65)}{\geqslant} C_{1} \tilde{n}^{-1 / m_{2}(G, H)} .
$$

Proof of Fact 28. Owing to the monotonicity of the 2-density and to the assumption on $G$ in Lemma 23, we have $m_{2}\left(G_{-}\right) \leqslant m_{2}(G)<x^{*}(H)$. Moreover, by our choice of constants, we have

$$
\widetilde{n} \stackrel{(65)}{\geqslant} \varrho n_{0} \stackrel{(50)}{\geqslant} n_{0}\left(G_{-}, H\right)
$$

and

$$
C\left(G_{-}, H\right) \tilde{n}^{-1 / m_{2}\left(G_{-}, H\right)} \stackrel{(43),(73)}{\lessgtr} p_{1} \stackrel{(31),(51)}{\lessgtr} \alpha n^{-1 / m^{*}(H)} \leqslant \widetilde{n}^{-1 / m^{*}(H)} .
$$

Thus we may apply the induction hypothesis to $G_{n, p_{1}}[V]$ to infer

$$
\mathbb{P}\left(G_{n, p_{1}} \in \neg \mathcal{A}_{V}\right) \leqslant 2^{-b\left(G_{-}, H\right) \tilde{n}^{v} H p_{1}^{e_{H}}(45),(51),(65)} 2^{-2 b_{1} n^{v} H p^{e_{H}}}
$$

recalling the definition of $\mathcal{A}_{V}$ in (68).

Proof of Fact 29. Consider a fixed graph $T \in \mathcal{T}$ as defined before (69), and let

$$
J:=G_{1-}^{H} \cap G_{2-}^{H}
$$

denote the intersection of the two graphs from $\mathcal{F}\left(G_{-}, H\right)$ forming $T$. We obtain with Lemma 10 that

$$
\widetilde{n}^{v(J)} p_{1}^{e(J)} \stackrel{(73)}{\geqslant} \widetilde{n}^{v(J)-e(J) / m_{2}(G, H)} \stackrel{\text { L.10 }}{\geqslant} \widetilde{n}^{2-L\left(G_{1-}^{H}\right)},
$$


where in the first step we also used that $C_{1} \geqslant 1$. Thus the expected number of copies of $T$ in $G_{n, p_{1}}[V]$ is bounded by

$$
\begin{aligned}
& \tilde{n}^{v(T)} p_{1}^{e(T)} \stackrel{(74)}{\lessgtr} \widetilde{n}^{v\left(G_{1-}^{H}\right)+v\left(G_{2-}^{H}\right)-2+L\left(G_{1-}^{H}\right)} p_{1}^{e\left(G_{1-}^{H}\right)+e\left(G_{2-}^{H}\right)} \\
& \stackrel{(8)}{=} \widetilde{n}^{2\left(v_{G}+\left(e_{G}-1\right)\left(v_{H}-2\right)\right)-2-L\left(G_{2-}^{H}\right)} p_{1}^{2\left(e_{G}-1\right) e_{H}} \\
& \leqslant \widetilde{n}^{2 v_{G}-2}\left(\widetilde{n}^{v_{H}-2} p_{1}^{e_{H}}\right)^{2\left(e_{G}-1\right)},
\end{aligned}
$$

where in the last step we used that $L\left(G_{2-}^{H}\right)$ is nonnegative. Thus in total the expected number of graphs from $\mathcal{T}$ in $G_{n, p_{1}}[V]$ is at most

$$
2^{\ell^{2}-1} \cdot \widetilde{n}^{2 v_{G}-2}\left(\widetilde{n}^{v_{H}-2} p_{1}^{e_{H}}\right)^{2\left(e_{G}-1\right)}
$$

where we bounded $|\mathcal{T}|$ by the number of graphs on at most $\ell$ vertices (recall (39)), which in turn is bounded by $\ell 2^{\left(\begin{array}{l}\ell \\ 2\end{array}\right)} \leqslant 2^{\ell^{2}-1}$.

Fact 29 now follows with Markov's inequality, recalling the definition of $\mathcal{D}_{V}$ in (69).

Proof of Fact 30. Consider a fixed set $V \subseteq[n],|V|=\widetilde{n}$, and an arbitrary graph $K \subseteq K_{n}$. For any red-blue-coloring $h$ of $E_{H}(K)$ and for every edge $e \in\left(\begin{array}{l}V \\ 2\end{array}\right)$, let

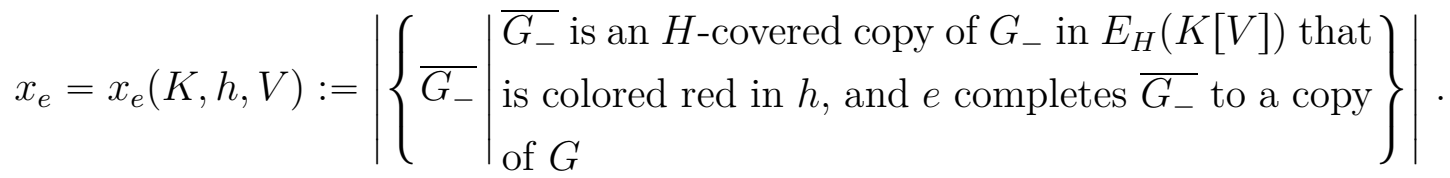

Note that, by our definition of $\Gamma(K, h, V)$ in (66)), for all $e \in \Gamma(K, h, V)$ we have

$$
x_{e} \geqslant z \stackrel{(56),(73)}{\geqslant} c_{\Gamma} C_{1}^{e_{H}\left(e_{G}-1\right)} n^{v_{G}-2}\left(n^{v_{H}-2} \widetilde{n}^{-e_{H} / m_{2}(G, H)}\right)^{e_{G}-1} \stackrel{(7),(43)}{\geqslant} 3
$$

where in the second inequality we also used that $\tilde{n} \leqslant n$.

We will show that if $K$ is in $\mathcal{A}_{V}$, we have

$$
\sum_{e \in \Gamma(K, h, V)} x_{e} \geqslant \frac{a\left(G_{-}, H\right)}{2} \cdot \tilde{n}^{v_{G}}\left(\widetilde{n}^{v_{H}-2} p_{1}^{e_{H}}\right)^{e_{G}-1}
$$

for every coloring $h$ of $E_{H}(K)$ that contains no blue copy of $H$, and that if $K$ is in $\mathcal{D}_{V}$, we have

$$
\sum_{e \in \Gamma(K, h, v)}\left(\begin{array}{c}
x_{e} \\
2
\end{array}\right) \leqslant 2^{\ell^{2}} \ell^{2 v_{G}} \cdot \widetilde{n}^{2 v_{G}-2}\left(\widetilde{n}^{v_{H}-2} p_{1}^{e_{H}}\right)^{2\left(e_{G}-1\right)}
$$

for every coloring $h$ of $E_{H}(K)$.

As by Jensen's inequality we have

$$
\sum_{e \in \Gamma(K, h, V)}\left(\begin{array}{c}
x_{e} \\
2
\end{array}\right) \geqslant|\Gamma(K, h, V)|\left(|\Gamma(K, h, V)|^{-1} \sum_{e \in \Gamma(K, h, V)} x_{e}\right) \stackrel{(75)}{2} \frac{\left(\sum_{e \in \Gamma(K, h, V)} x_{e}\right)^{2}}{3|\Gamma(K, h, V)|},
$$


(where in the second inequality we used that $\left(\begin{array}{l}x \\ 2\end{array}\right) \geqslant x^{2} / 3$ for $x \geqslant 3$ ), (76) and (77) will imply that for any $K \in \mathcal{A}_{V} \cap \mathcal{D}_{V}$ we have

$$
|\Gamma(K, h, V)| \stackrel{(78)}{\geqslant} \frac{\left(\sum_{e \in \Gamma(K, h, V)} x_{e}\right)^{2}}{3 \sum_{e \in \Gamma(K, h, V)}\left(\begin{array}{c}
x_{e} \\
2
\end{array}\right)} \stackrel{(76),(77)}{\geqslant} \frac{a\left(G_{-}, H\right)^{2}}{12 \cdot 2^{\ell^{2}} \cdot \ell^{2 v_{G}}} \cdot \tilde{n}^{2} \stackrel{(40)}{=} d \widetilde{n}^{2}
$$

for every coloring $h$ of $E_{H}(K)$ that does not contain a blue copy of $H$, i.e., that $K$ satisfies $\mathcal{B}_{V}$ as defined in (67).

It remains to show (76) and (77). To verify (76), recall that $k_{G_{-}}(K, h, V)$ denotes the total number of $H$-covered red copies of $G_{-}$in $E_{H}(K[V])$. Since every such copy contributes to at least one of the $x_{e}$, we have

$$
\sum_{e \in\left(\begin{array}{c}
V \\
2
\end{array}\right)} x_{e} \geqslant k_{G_{-}}(K, h, V) .
$$

Note that

$$
z \stackrel{(56)}{=} c_{\Gamma} \cdot n^{v_{G}-2}\left(n^{v_{H}-2} p_{1}^{e_{H}}\right)^{e_{G}-1} \stackrel{(42),(65)}{\lessgtr} a\left(G_{-}, H\right) \cdot \widetilde{n}^{v_{G}-2}\left(\widetilde{n}^{v_{H}-2} p_{1}^{e_{H}}\right)^{e_{G}-1} .
$$

Since by definition of $\Gamma(K, h, V)$ we have $x_{e}<z$ for all $e \in\left(\begin{array}{c}V \\ 2\end{array}\right) \backslash \Gamma(K, h, V)$ (recall (66)), it follows that

$$
\begin{aligned}
\sum_{e \in \Gamma(K, h, V)} x_{e} \stackrel{(79)}{\geqslant} k_{G_{-}}(K, h, V)- & \sum_{e \in\left(\begin{array}{c}
V \\
2
\end{array}\right) \backslash \Gamma(K, h, V)} x_{e} \geqslant k_{G_{-}}(K, h, V)-\left(\begin{array}{l}
\tilde{n} \\
2
\end{array}\right) \cdot z \\
& \stackrel{(80)}{\geqslant} k_{G_{-}}(K, h, V)-\frac{a\left(G_{-}, H\right)}{2} \cdot \tilde{n}^{v_{G}}\left(\widetilde{n}^{v_{H}-2} p_{1}^{e_{H}}\right)^{e_{G}-1} .
\end{aligned}
$$

It follows from the definition of $\mathcal{A}_{V}$ in (68) that indeed (76) holds for every coloring $h$ of $E_{H}(K)$ that contains no blue copy of $H$ if $K$ is in $\mathcal{A}_{V}$.

To verify (77), recall that every $H$-covered copy of $G_{-}$is contained in a copy of a graph $G_{-}^{H} \in \mathcal{F}\left(G_{-}, H\right)$ (see Definition 8 and Definition 9). It follows with the definition of $k_{\mathcal{T}}(K, V)$ (see the paragraph before $(69)$ ) that

$$
\sum_{e \in\left(\begin{array}{c}
V \\
2
\end{array}\right)}\left(\begin{array}{c}
x_{e} \\
2
\end{array}\right) \leqslant \ell^{2 v_{G}} k_{\mathcal{T}}(K, V)
$$

where $\ell$ is as defined in (39). Here the constant $\ell^{2 v_{G}}$ follows from the fact that a given copy of some $T \in \mathcal{T}$ contributes at most $\left(\left(v_{T}\right)_{v_{G}}\right)^{2} \leqslant\left(v_{T}\right)^{2 v_{G}} \leqslant \ell^{2 v_{G}}$ to the sum.

Consequently, if $K \in \mathcal{D}_{V}$, the definition of $\mathcal{D}_{V}$ in (69) implies that

$$
\sum_{e \in \Gamma(K, h, v)}\left(\begin{array}{c}
x_{e} \\
2
\end{array}\right) \leqslant \sum_{e \in\left(\begin{array}{c}
V \\
2
\end{array}\right)}\left(\begin{array}{c}
x_{e} \\
2
\end{array}\right) \stackrel{(69),(81)}{\leftarrow} 2^{\ell^{2}} \ell^{2 v_{G}} \cdot \widetilde{n}^{2 v_{G}-2}\left(\widetilde{n}^{v_{H}-2} p_{1}^{e_{H}}\right)^{2\left(e_{G}-1\right)}
$$

for every coloring $h$ of $E_{H}(K)$, as claimed in (77). 
3.3. Proof of Claim 27. It now remains to prove Claim 27.

Proof of Claim 27. Consider a fixed graph $K \in \mathcal{B}$ and a fixed red-blue-coloring $h$ of $E_{H}(K)$. By definition of the event $\mathcal{B}$ (recall $(58)$ ), the graph $\Gamma(K, h)$ is $(\varrho, d)$-dense.

Note that due to (44) and (52) we have

$$
b^{\prime} n^{v_{H}} p_{2}^{e_{H}} \geqslant\left(b^{\prime} / 2\right) n^{v_{H}} p^{e_{H}} .
$$

Thus Lemma 22 yields with (31) and our choice of constants in (41) and (50) that with probability at least

$$
1-2^{1-b^{\prime} n^{v} H} p_{2}^{e_{H}} \stackrel{(82),(46)}{\geqslant} 1-2^{1-2 b_{2} n^{v} H p^{e} H} \geqslant 1-2^{-b_{2} n^{v_{H}} p^{e_{H}}}
$$

(where in the last step we used that $1 \leqslant b^{v_{H}} p^{e_{H}} \leqslant b_{2} n^{v_{H}} p^{e_{H}}$ due to (53) and (48)), the graph $\Gamma(K, h) \cap G_{n, p_{2}}$ contains a family of at least $b^{\prime} n^{v_{H}} p_{2}^{e_{H}}$ pairwise edge-disjoint copies of $H$.

To avoid creating a blue copy of $H$, one edge from each of these copies needs to be colored red, and by the definition of $\Gamma(K, h)$ (see $(57)$ ), each such edge that is colored red creates at least $z$ many $H$-covered red copies of $G$. Thus any extension of $h$ to a coloring of $E_{H}(K) \cup E_{H}\left(G_{n, p_{2}}\right) \subseteq E_{H}\left(K \cup G_{n, p_{2}}\right)$ creates a blue copy of $H$ or at least

$$
\begin{gathered}
b^{\prime} n^{v_{H}} p_{2}^{e_{H}} \cdot z \stackrel{(56),(82)}{\stackrel{(}{\geqslant}} b^{\prime} / 2 \cdot c_{\Gamma} \cdot n^{v_{H}} p^{e_{H}} \cdot n^{v_{G}-2}\left(n^{v_{H}-2} p_{1}^{e_{H}}\right)^{e_{G}-1} \\
\stackrel{(47),(51)}{=} a n^{v_{G}}\left(n^{v_{H}-2} p^{e_{H}}\right)^{e_{G}}
\end{gathered}
$$

many $H$-covered red copies of $G$. Thus $G_{n, p_{2}}$ is indeed in $\mathcal{A}_{K, h}$ as defined in (55) with the claimed probability.

\section{§4. Concluding REMARKS}

We believe that the proof for the two-color case given here can be extended to the setting with more than two colors along the lines of [21]. Namely, for given graphs $H_{1}, \ldots, H_{k}$ with $m_{2}\left(H_{k}\right) \leqslant \cdots \leqslant m_{2}\left(H_{2}\right)<m_{2}\left(H_{1}\right)$ and $H_{1}$ strictly balanced w.r.t. $d_{2}\left(H_{2}, \cdot\right)$, one should be able to prove that $\mathbb{P}\left(G_{n, p} \rightarrow\left(H_{1}, \ldots, H_{k}\right)\right)=1-o(1)$ if $p \geqslant C n^{-1 / m_{2}\left(H_{2}, H_{1}\right)}$ as follows: Clearly, in order to prove $G_{n, p} \rightarrow\left(H_{1}, \ldots, H_{k}\right)$ it suffices to prove $G_{n, p} \rightarrow(G, \ldots, G, H)$, where $G$ denotes the disjoint union of $H_{2}, \ldots, H_{k}$, and $H:=H_{1}$. Furthermore, it is not hard to see that $m_{2}(G)=m_{2}\left(H_{2}\right)$, and consequently also $m_{2}(G, H)=m_{2}\left(H_{2}, H_{1}\right)$. Thus it suffices to show that $\mathbb{P}\left(G_{n, p} \rightarrow(G, \ldots, G, H)\right)=1-o(1)$ if $p \geqslant C n^{-1 / m_{2}(G, H)}$. We believe that this can be done by combining the approach via double induction (on $e(G)$ and the number of colors $k$ ) used in [21] with the ideas presented in this paper. Note that this implies using Lemma 1 of [21], which relies on the regularity lemma for dense graphs. 
We do not pursue this further here. In our view, a more interesting next step would be to extend the approach taken in [7] to the asymmetric scenario, with the goal of deriving 1-statements for more general settings, in particular for the hypergraph setting. This might also help in getting rid of the balancedness assumption on $H$ in the existing proofs.

An altogether different open question is the proof of the 0-statement in Conjecture 3. With some extra work the approach in [19] can be pushed through to prove the 0-statement

for certain graphs $G$ and $H$ that are not complete, but a general proof does not seem to be within reach of the known methods.

\section{REFERENCES}

[1] C. Berge, Graphs and hypergraphs, revised, North-Holland Publishing Co., Amsterdam, 1976. Translated from the French by Edward Minieka, North-Holland Mathematical Library, Vol. 6. MR0384579 (52 \#5453) $\uparrow 2.5$

[2] S. A. Burr, P. Erdős, and L. Lovász, On graphs of Ramsey type, Ars Combinatoria 1 (1976), no. 1, 167-190. MR0419285 (54 \#7308) $\uparrow 1.3$

[3] F. Chung and R. Graham, Erdös on graphs, A K Peters Ltd., Wellesley, MA, 1998. His legacy of unsolved problems. MR1601954 (99b:05031) $\uparrow 1.2$

[4] D. Conlon and W. T. Gowers, Combinatorial theorems in sparse random sets. Submitted. $\uparrow 1.1$

[5] C. M. Fortuin, P. W. Kasteleyn, and J. Ginibre, Correlation inequalities on some partially ordered sets, Comm. Math. Phys. 22 (1971), 89-103. MR0309498 (46 \#8607) $\uparrow 2.4$

[6] P. Frankl and V. Rödl, Large triangle-free subgraphs in graphs without $K_{4}$, Graphs Combin. 2 (1986), no. 2, 135-144. MR932121 (89b:05124) $\uparrow 1.1$

[7] E. Friedgut, V. Rödl, and M. Schacht, Ramsey properties of random discrete structures, Random Structures Algorithms 37 (2010), no. 4, 407-436. MR2760356 ^1.1, 1.4, 4

[8] S. Gerke and A. Steger, The sparse regularity lemma and its applications, Surveys in combinatorics 2005, 2005, pp. 227-258. MR2187740 ^1.2

[9] T. E. Harris, A lower bound for the critical probability in a certain percolation process, Proc. Cambridge Philos. Soc. 56 (1960), 13-20. MR0115221 (22 \#6023) ^1.4, 2.4, 17

[10] S. Janson, Poisson approximation for large deviations, Random Structures \& Algorithms 1 (1990), no. 2, 221-229. MR1138428 (93a:60041) $\uparrow 18$

[11] S. Janson, T. Łuczak, and A. Rucinski, Random graphs, Wiley-Interscience Series in Discrete Mathematics and Optimization, Wiley-Interscience, New York, 2000. MR1782847 (2001k:05180) 个1.1, 1.3, 1.4

[12] S. Janson and A. Ruciński, The deletion method for upper tail estimates, Combinatorica 24 (2004), no. 4, 615-640. MR2096818 (2005i:60019) $\uparrow 1.4$

[13] Y. Kohayakawa, Szemerédi's regularity lemma for sparse graphs, Foundations of computational mathematics (rio de janeiro, 1997), 1997, pp. 216-230. MR1661982 (99g:05145) ^1.2

[14] Y. Kohayakawa and B. Kreuter, Threshold functions for asymmetric Ramsey properties involving cycles, Random Structures \& Algorithms 11 (1997), no. 3, 245-276. MR1609513 (99g:05159) ^2, 1.2, $3,1.2,1.3$ 
[15] Y. Kohayakawa, T. Łuczak, and V. Rödl, On $K^{4}$-free subgraphs of random graphs, Combinatorica 17 (1997), no. 2, 173-213. MR1479298 (98h:05166) $\uparrow 1.2$

[16] Y. Kohayakawa and V. Rödl, Regular pairs in sparse random graphs. I, Random Structures Algorithms 22 (2003), no. 4, 359-434. MR1980964 (2004b:05187) 11.2

[17] B. Kreuter, Threshold functions for asymmetric Ramsey properties with respect to vertex colorings, Random Structures \&Algorithms 9 (1996), no. 3, 335-348. MR1606853 (99c:05178) ^1.2, 2.5

[18] T. Łuczak, A. Ruciński, and B. Voigt, Ramsey properties of random graphs, J. Combin. Theory Ser. B 56 (1992), no. 1, 55-68. MR1182457 (94b:05172) $\uparrow 1.1,1.1$

[19] M. Marciniszyn, J. Skokan, R. Spöhel, and A. Steger, Asymmetric Ramsey properties of random graphs involving cliques, Random Structures Algorithms 34 (2009), no. 4, 419-453. MR2531778 (2010i:05313) $\uparrow 1.2,1.3,4$

[20] V. Rödl and A. Ruciński, Lower bounds on probability thresholds for Ramsey properties, Combinatorics, Paul Erdős is eighty, vol. 1, 1993, pp. 317-346. MR1249720 (95b:05150) ^1.1, 1.1, 1

[21] — Threshold functions for Ramsey properties, J. Amer. Math. Soc. 8 (1995), no. 4, 917-942. MR1276825 (96h:05141) $11.1,1,1.3,1.4,2.4,3.1,3.2,4$

[22] — Ramsey properties of random hypergraphs, J. Combin. Theory Ser. A 81 (1998), no. 1, 1-33. MR1492867 (98m:05175) $\uparrow 1.1,1.4$

[23] V. Rödl, A. Ruciński, and M. Schacht, Ramsey properties of random k-partite, $k$-uniform hypergraphs, SIAM J. Discrete Math. 21 (2007), no. 2, 442-460 (electronic). MR2318677 (2008d:05103) ^1.1, 1.4

[24] M. Schacht, Extremal results for random discrete structures. Submitted. $\uparrow 1.4$

Instituto de Matemática e Estatística, Universidade de São Paulo, São Paulo, Brazil E-mail address: yoshi@ime.usp.br

Fachbereich Mathematik, Universität Hamburg, Hamburg, Germany

E-mail address: schacht@math.uni-hamburg.de

Algorithms \& Complexity Group, Max Planck Institute for Informatics, SaArbrücken, GERMANy

E-mail address: rspoehel@mpi-inf.mpg.de 